\title{
1 Structural variants in Chinese population and their impact on 2 phenotypes, diseases and population adaptation
}

3

4 Zhikun $\mathrm{Wu}^{1 \#}$, Zehang Jiang ${ }^{1 \#}$, Tong $\mathrm{Li}^{1 \#}$, Chuanbo $\mathrm{Xie}^{2+}$, Liansheng Zhao ${ }^{3,4+}$, Jiaqi Yang ${ }^{1}$, Shuai Ouyang ${ }^{1}$,

$5 \quad$ Yizhi Liu' ${ }^{1}, \mathrm{Tao}^{3} \mathrm{Li}^{3 *}$, Zhi Xie ${ }^{1 *}$

$6{ }^{1}$ State Key Laboratory of Ophthalmology, Zhongshan Ophthalmic Center, Sun Yat-sen University,

7 Guangzhou, China

$8 \quad{ }^{2}$ Sun Yat-sen University Cancer Center, Sun Yat-sen University, Guangzhou, China

$9{ }^{3}$ Mental Health Center and Psychiatric Laboratory, the State Key Laboratory of Biotherapy, West China

10 Hospital of Sichuan University, Chengdu, China

$11{ }^{4}$ Guangdong-Hong Kong-Macao Greater Bay Area Center for Brain Science and Brain-Inspired

12 Intelligence, Guangzhou, China

$13 \quad{ }^{\#+}$ Equally contributed.* Email: xiezhi@gmail.com, litaohx@scu.edu.cn 


\section{Summary}

15 A complete characterization of genetic variation is a fundamental goal of human genome research. Long-read sequencing (LRS) improves the sensitivity for structural variant (SV) discovery and facilitates a better understanding of the SV spectrum in human genomes. Here, we conduct the first LRS-based SV analysis in Chinese population. We perform whole-genome LRS for 405 unrelated Chinese, with 68 phenotypic and clinical measurements. We discover a complex landscape of 132,312 non-redundant SVs, of which 53.3\% are novel. The identified SVs are of high-quality validated by the PacBio high-fidelity sequencing and PCR experiments. The total length of SVs represents approximately $13.2 \%$ of the human reference genome. We annotate 1,929 loss-of-function SVs affecting the coding sequences of 1,681 genes. We discover new associations of SVs with phenotypes and diseases, such as rare deletions in $H B A 1 / H B A 2 / H B B$ associated with anemia and common deletions in $G H R$ associated with body height. Furthermore, we identify SV candidates related to human immunity that differentiate sub-populations of Chinese. Our study reveals the complex landscape of human SVs in unprecedented detail and provides new insights into their roles contributing to phenotypes, diseases and evolution. The genotypic and phenotypic resource is freely available to the scientific community.

\section{Introduction}

33 Human genetic variants comprise single-nucleotide variants (SNVs), small insertions or deletions (InDels) and structural variants (SVs), and profoundly contribute to many physical traits and human diseases. SVs are defined as genomic rearrangements that range from 50 base-pairs $(\mathrm{bp})$ to over megabases $(\mathrm{Mbs})$ in length, including different forms such as deletion (DEL), insertion (INS), duplication (DUP) and inversion (INV) ${ }^{1}$.

38 Accumulating evidence has demonstrated that SVs are associated with many human diseases, such as neurodevelopment disease and cancer ${ }^{2-6}$. on short-read sequencing (SRS) technologies, the discovery and genotyping of SVs 
42 have been hampered due to the limited power of SRS to detect SVs that frequently

43 occur in repetitive regions with complex structures. Consequently, the current human

44 reference genome contains a comprehensive map of SNVs, InDels, but only a relatively small number of $\mathrm{SVs}^{7}$. More recently, third-generation sequencing (TGS) platforms such as Pacific Biosciences (PacBio) and Oxford Nanopore Technologies (ONT) provide long-read sequencing (LRS), which improves the sensitivity for SV discovery and facilitates a better understanding of the SV spectrum in human genomes ${ }^{8,9}$.

Recently, a milestone study generated 15 human genomes using LRS with PacBio technology ${ }^{8}$. Despite the small sample size, the authors discovered 99,604 nonredundant SVs and 2,238 SVs were shared by all 15 genomes. More recently, a population-scale SV study using LRS was reported on an Icelandic population ${ }^{10}$. The authors identified a median of 23,111 SVs in each sample and some of them might be Icelander specific. Icelanders are a North Germanic ethnic group that is historically coherent, with an estimated population close to 360,000 . In contrast, Han Chinese is the largest ethnic group in the world, with a total population of more than 1.3 billion, comprising around $19 \%$ of the human population. China has an area of 9.6 million $\mathrm{km}^{2}$, with two mega-regions, North and South China. Although several recent studies have reported on SVs in Chinese genomes using $\operatorname{LRS}^{9,11-13}$, none of them depicted multiple genomes. The complexity and diversity of genetic variation characterized by SVs among Chinese populations are unclear.

In order to gain a more complete view of human genomes, we genotyped Chinese population by performing whole-genome LRS of 405 unrelated Chinese individuals, with 68 phenotypic and clinical measurements. We detected 132,312 non-redundant SVs, of which $53.3 \%$ were novel. The identified SVs were of high-quality validated by the PacBio high fidelity (HiFi) sequencing and PCR experiments. We showed the global properties of the SVs and their widespread phenotypic, clinical and evolutionary impacts. To sum, we present an important resource for human genome research and precision medicine. Our study reveals the complex landscape of human SVs in 
unprecedented detail and provides new insights into their roles contributing to phenotypes, diseases and evolution. The genotypic and phenotypic resource is freely available to the scientific community.

\section{Results}

\section{Sequencing, SV discovery and validation}

We performed whole-genome LRS for 405 unrelated Chinese via the PromethION platform (ONT). Among all the individuals, 206 (50.9\%) were males and 199 (49.1\%) were females. The ages ranged from 22 to 81 years, with a median age of 42 years. These individuals were from 18 provinces in the North (124 individuals), South (198) and Southwest (53) of China, and the ancestral regions of 30 individuals were not known (Fig. 1a and Supplementary Table 1). Among them, 68 phenotypic and clinical measurements for 327 individuals were obtained by health screening (Supplementary Table 2).

Resequencing of these 405 Chinese generated a total of 20.7 terabases $(\mathrm{Tb})$ of cleaned sequences, with an average of 51.0 gigabases $(\mathrm{Gb})$ per individual, representing an average depth of approximate 17-fold (Supplementary Fig. 1a and Supplementary Table 1). The cleaned reads were then mapped onto the human reference genome GRCh38, and base mapping rate for different individuals varied from $89.0 \%$ to $96.2 \%$, with an average of $94.1 \%$ (Fig. 1b). The mean error rate was $12.6 \%$, ranging from $10.8 \%$ to $16.0 \%$ (Methods), which was lower than that of a recent study $(15.2 \%)^{10}$ and similar to a prior benchmarking study $(12.6 \%)^{14}$. The percent of deletions, insertions and substitutions (mismatches) was 5.1\%, 3.4\% and 4.1\%, respectively (Fig. 1c).

Four classes of canonical SVs (DEL, INS, DUP and INV) with a length of at least 50-bp were detected. To obtain reliable SVs, we used three SV callers, Sniffles ${ }^{15}$, NanoVar ${ }^{16}$ and NanoSV ${ }^{17}$, all of which were specifically designed for SV detection from LRS (Supplementary Fig. 2a). We retained the SVs identified by at least two callers (Fig. 1d), which could effectively reduce the false positive of SV detection, 
97 particularly for sequencing data with lower depth (Supplementary Fig. 2b and

98 Methods). In addition, we applied three filtering steps that removed an average of

99 1,331 SVs per sample to further reduce unreliable SVs (Fig. 1e, Supplementary Table

1003 and Methods). Finally, we identified 18,489 high-confidence SVs per sample,

101 ranging from 15,439 to 22,505 (Fig. 1f and Supplementary Table 3). The numbers of

102 SVs followed an approximately normal distribution (Supplementary Fig. 2c). DELs

103 and INSs were predominant, and each sample contained an average of 8,215 DELs

104 (44.4\%), 9,942 INSs (53.8\%), 258 DUPs (1.4\%) and 74 INVs (0.4\%) (Fig. 1f). A

105 balanced number between DEL and INS was also observed in the previously LRS-

106 based SV studies ${ }^{8,10}$, and slightly higher ratio of INSs than DELs may be due to the

107 DEL bias of GRCh38 $8^{18}$.

108 We estimated the relationship between the SV number and sequencing depth. It

109 was observed that the number of SVs just slightly increased when the depth was more

110 than 15-fold (Supplementary Fig. 2b). The number of SVs was around 19,070 per

111 sample at 15-fold depth and that increased to around 20,378 at 40-fold depth

112 (Supplementary Fig. 2b), suggesting that 15-fold sequencing was effective to detect

113 SVs for a population-scale study.

114 It is known that LRS technology such as ONT with high sequence error more likely

115 leads to mis-mapping against the reference genome and therefore causes higher false

116 discovery of SVs. To estimate the false discovery rate (FDR) of SVs using our SV

117 identification strategy, SVs detected from a 15-fold ONT dataset (HG002, the child)

118 were validated by a PacBio high-fidelity (HiFi) dataset from a parent-offspring trio in

119 Genome in a Bottle (GIAB), whose base accuracy was up to $99.8 \%{ }^{19}$. Out of the 18,737

120 detected SVs for the HG002 dataset, the overall FDR was 3.2\%, illustrating the

121 reliability of SVs detected using our SV identification strategy from ONT reads with

122 15-fold depth (Methods). INVs are generally enriched for false positives. To estimate

123 FDR for INVs, we further manually investigated the strand-specific alignment of long-

124 read for the INV region using Integrative Genomics Viewer $(\mathrm{IGV})^{20}$. We checked 75 
125 INVs and detected four false positives with an estimated FDR of 5.3\%.

\section{Comparison with published SV datasets}

127 We merged the SVs detected from all the samples for each SV type and constructed a 128 set of 132,312 non-redundant SVs, comprising 67,405 DELs, 60,182 INSs, 3,956 DUPs

129 and 769 INVs (Fig. 2a). We compared our data with four previously published datasets

130 generated using either NGS or TGS platforms (Fig. 2b). To note, the LRS15 set was 131 the only one generated using the TGS platform (PacBio) from 15 individuals $^{8}$, which

132 had the largest overlaps with our data $(38,963)$ and had the smallest unique SV set of 133 its own $(60,641)$. For other three datasets generated using the NGS platform, there were $13430,783,24,741$ and 24,472 SVs that overlapped with the Database of Genomic Variants $135(\mathrm{DGV})^{21}$, Genome Aggregation Database (gnomAD) ${ }^{22}$ and the Human Genome 136 Diversity panel (HGD) $)^{23}$, respectively. In total, 70,471 (53.3\%) SVs from our data have 137 not been previously reported. We further examined the recovery of SVs in the previous 138 datasets for each SV type. It was notable that although the total numbers of INSs and

139 DELs were similar in our dataset, the number of recovered INSs with the LRS-based 140 study, LRS15, was much larger than the SRS-based datasets, illustrating that LRS 141 technology is particularly efficient to detect INSs (Fig. 2c).

\section{Genomic features of SVs}

143 SVs were nonrandomly distributed across the chromosome and the number of SVs

144 significantly correlated with chromosome length $\left(R=0.92, P=8.3 \times 10^{-10}\right.$, Pearson 145 correlation test) (Fig. 2d). The number generally increased at the ends of chromosome 146 arms, particularly for DELs, INSs and DUPs (Fig. 2e). The sub-telomeric bias of the 147 long arms of chromosomes was higher compared to that of short arms (Supplementary

148 Fig. 3), which was in accord with the pattern detected by an LRS-based SV study using 149 a PacBio platform ${ }^{8}$.

150 We observed that the median lengths of INSs and DELs were $111 \mathrm{bp}$ and $120 \mathrm{bp}$, 151 respectively, which was significantly shorter than that of DUPs (1,081 bp) and INVs $152(5,496 \mathrm{bp})$ (Fig. 2f). The longer length of DUPs and INVs was confirmed by our PacBio 
153 HiFi datasets as well as the PacBio HiFi datasets of the trio from GIAB, indicating that

154 the observation was not the ONT platform specific. The numbers of DELs and INSs

155 rapidly decreased as their length increased. There were two clear peaks at sizes around

$156300 \mathrm{bp}$ and 6 kilobases (kb) for both DELs and INSs (Supplementary Fig. 4),

157 corresponding to short interspersed nuclear elements (SINEs) and long interspersed 158 nuclear elements (LINEs) $)^{8,10}$.

159 The total length of non-redundant SVs was $395.6 \mathrm{Mb}$, representing approximately

$16013.2 \%$ of the human reference genome, including 125.7 Mb of DELs, $19.8 \mathrm{Mb}$ of INSs,

$161 \quad 104.8 \mathrm{Mb}$ of DUPs and 145.2 Mb of INVs (Fig. 2g). On average, SVs affected 23.0 Mb

162 (around $0.8 \%$ ) of the genome per individual (Supplementary Table 4), where the 163 average lengths of DELs and INSs were $7.2 \mathrm{Mb}(31.2 \%$ of the total SV length) and 3.7

$164 \mathrm{Mb}(15.9 \%)$, respectively. Despite their lower number, INVs (9.6 Mb, 41.7\%) and

165 DUPs $(2.5 \mathrm{Mb}, 11.1 \%)$ contributed equivalently to the total SV length due to their

166 considerably longer length. Same INSs were more common in individuals compared to

167 those of other types, which may be partly due to the DELs bias of GRCh38 or

168 purification selection of INSs in Chinese population (Fig. 2h). 75.4\% of SVs contained

169 repeat sequences (Supplementary Table 5), which was consistent with the knowledge

170 that SVs tended to occur in segments with more repetitive sequences ${ }^{1}$. Among all of

171 these repeats, VNTRs (25.1\% of SVs) and SINEs (19.7\%) were predominant

172 (Supplementary Table 5).

\section{Allele frequencies of SVs}

174 Our datasets offer us an opportunity to identify SVs with a low frequency in a 175 population. We grouped the SVs into four categories based on their allele frequencies 176 (AF): singleton (allele count $=1)$, rare (allele count $>1$ and $\mathrm{AF} \leq 0.01)$, low $(0.01<\mathrm{AF}$ $177 \leq 0.05)$ and common $(\mathrm{AF}>0.05)$. Singletons $(56,239)$ represented $42.5 \%$ of the total 178 identified SVs (Fig. 3a and Supplementary Fig. 5). Additionally, there were 28,925 179 rare (21.9\%), 14,296 low (10.8\%) and 32,852 common (24.8\%) SVs. Among the 180 common SVs, 1,264 (3.9\%) were shared in all samples. The lower AF values of an 
181 identified SV, the larger proportion of novel SVs that were not previously reported (Fig.

182 3a). Specifically, $75.1 \%$ of singleton SVs were novel, which was similar to the 183 percentage of novel singleton SNVs (72.8\%) in Koreans ${ }^{24}$. In contrast, $14.9 \%$ of 184 common SVs were not previously reported.

185 Singleton SVs are prone to false positives compared with other categories because 186 they are detected from one sample. To validate the accuracy of singletons, we designed 187 the primers for 154 randomly selected singleton DELs and INSs from 20 samples and 188 validated 145 by PCR experiments $($ FDR $=5.8 \%)$. (Supplementary Table 6). In 189 addition, we sequenced four samples in our study using the Pacbio HiFi (average depth 190 of 9.35-fold, Methods). There were 510 singletons detected from the ONT reads for 191 these four samples. And we found that $32 \mathrm{SVs}$ were false positive based on the 192 validation of PacBio HiFi reads and manual investigation by IGV (FDR $=6.3 \%$ ).

193 To estimate how much SV spectrum in Chinese population has been detected in 194 our study, we assessed the SV numbers with different categories as the number of 195 samples increased through multiple sampling. Relatively stable number of the common 196 and low SVs indicated that the samples used in this study was sufficient to characterize 197 these SVs (Supplementary Fig. 6). The continued increasing trends of the singleton 198 and rare SVs suggested that a larger sample size was needed to sufficiently detect SVs 199 with an $\mathrm{AF} \leq 0.01$.

\section{Functional relevance of SVs}

201 To explore their potential functions, we annotated SVs based on their genomic location,

202 including coding sequence (CDS), untranslated region (UTR), promoter and intron. A 203 substantial amount (37.6\%) of SVs were located in introns, while $1.0 \%, 0.9 \%$ and $1.7 \%$ 204 of SVs were located in the promoter, UTR and CDS, respectively (Table 1). Among all 205 the SVs located in the UTR and CDS, singletons were significantly enriched compared 206 with the other categories $\left(P=1.1 \times 10^{-4}\right.$ for singletons in UTR and $5.8 \times 10^{-16}$ for 207 singletons in CDS, Fisher's exact test, Fig. 3b), suggesting that singleton SVs were 208 more likely to have genetic functions. 
We further classified the SVs that interacted with CDS into three subgroups

210 according to their breakpoint locations: predicted loss-of-function ( $\mathrm{pLoF})$, whole-gene

211 duplication (WDUP) and whole-gene inversion (WINV) (Methods). While pLoFs

212 delete coding nucleotides or alter open-reading frames, WDUPs generally cause a

213 copy-gain of an entire gene, and WINVs regulate gene expression through affecting the

214 position and order of upstream enhancers and genes ${ }^{25}$. We annotated a total of 2,277

215 SVs affecting the coding regions of 3,176 distinct genes, including 1,929 pLoF SVs,

216 affecting the CDS of 1,681 genes, as well as 202 WDUPs and 146 WINVs, covering

217581 and 1,331 genes, respectively (Fig. 3c). Interestingly, Gene Ontology (GO)

218 analysis revealed that there were 38 genes interested with pLoF SVs that were

219 significantly enriched in "immunoglobulin receptor binding" (odds ratio $=5.7$, adjusted

$220 \quad$ P value $=7.2 \times 10-18$, Benjamini-Hochberg corrected, Supplementary Fig. 7).

On average, individuals carry 2.7 and $2.9 \mathrm{pLoF}$ SVs for singleton and rare categories, respectively. More than half of pLoF SVs (57.6\%) were singletons, and the median length of all pLoF SVs was $2.2 \mathrm{~kb}$ (Fig. 3d). The median length of common pLoF SVs was just $251 \mathrm{bp}$, while that of singleton pLoF SVs was up to $4,480 \mathrm{bp}$

(Supplementary Fig. 8). Longer SVs in the singletons may be more likely to disrupt gene function.

\section{Phenotypic and clinical impacts of SVs}

228 In order to better understand how pLoF SVs impact clinical phenotypes and diseases, we annotated these SVs and their associated genes using the Genome-Wide Association 230 Studies (GWAS) catalog ${ }^{26}$, Online Mendelian Inheritance in Man (OMIM) ${ }^{27}$ and

231 Catalogue Of Somatic Mutations In Cancer (COSMIC) $)^{28}$. For a total number of 1,929 232 pLoF SVs, 1,231 (63.8\%) were intersected with genes cataloged in above databases 233 (Supplementary Table 7). Among the 1,231 SVs, 58.1\% to $60.2 \%$ belonged to the 234 singleton category (Fig. 4a), which was consistent with our previous enrichment 235 analysis showing that singletons were more likely to be functional (Fig. 3b). At the 236 gene level, all of the 1,929 pLoF SVs intersected with 1,681 distinct genes, where 957 
genes $(56.9 \%)$ were annotated in the three databases (Fig. 4b and Supplementary

\section{Table 7).}

239 Many phenotypically and clinically relevant SVs could be discovered from our 240 dataset. For example, we detected plausible causal variants associated with anemia

241 which has not been reported. We found a heterozygous rare DEL of $19.3 \mathrm{~kb}$ in three 242 individuals, covering the genes Hemoglobin Subunit Alpha 1 and 2 (HBA1 and $H B A 2$ ),

243 whose dysfunctions are known to cause $\alpha$-thalassemia ${ }^{29}$ (Fig. $4 \mathbf{c}$ ). In addition, one 244 individual had a heterozygous DEL of $27.4 \mathrm{~kb}$, containing gene Hemoglobin Subunit 245 Beta $(H B B)$, whose dysfunction is known to cause serious hemoglobinopathies, such 246 as sickle cell anemia and $\beta$-thalassemia (Fig. $4 \mathbf{c})^{30}$. As expected, the mean corpuscular 247 hemoglobin values (MCH, ranging from 21.3 to $23.8 \mathrm{pg}$ ) and the mean corpuscular 248 volume values (MCV, ranging from 65.9 to $72.3 \mathrm{fL}$ ) of these four individuals carrying 249 the aforementioned heterozygous DELs were significantly lower than individuals with 250 the reference allele in our study $(P=0.0006$ and 0.0005 for $\mathrm{MCH}$ and $\mathrm{MCV}$, 251 respectively, $t$-test corrected by FDR, Fig. 4c).

252 Besides rare SVs, we could also detect common SVs that were associated with 253 various phenotypes. We observed a DEL of $2.4 \mathrm{~kb}$, which existed in 35 homozygous 254 (15 males and 20 females) and 67 heterozygous (35 males and 32 females) carriers in 255 our study, covering the complete region of the third exon of GHR (Growth Hormone 256 Receptor), whose missense mutation is known to cause short stature and dwarfism (Fig. 257 4d $)^{31}$. Interestingly, the homozygous carriers were significantly shorter than 258 heterozygous carriers and people with homozygous reference allele for males (168.9 259 vs. $172.9(\mathrm{~cm})$ and 168.9 vs. $172.4(\mathrm{~cm}), P=0.033$ for both, $t$-test corrected by FDR, 260 Fig. 4d), suggesting that the DEL acted as recessive allele for this gene. In contrast, no 261 significant difference was observed between these three genotypes in females. The 262 growth hormone (GH)-insulin-like growth factor (IGF)-I axis is the principle endocrine 263 system regulating linear growth in children ${ }^{32}$. This sex different phenotype could be 264 caused by the cross-talk between the GH/IGF axis and sex hormones, where pituitary 
265 GH secretion regulates many sex-dependent genes in the liver ${ }^{33}$, and the pituitary is the

266 major source of IGF that determines body height, in a sex-dependent manner ${ }^{34}$.

267 We further conducted Genome-wide association study (GWAS) for the clinical 268 phenotypes based on genotyped SVs with minor allele frequency (MAF) $>0.05$. We 269 found that $25 \mathrm{SVs}$ were significantly associated with 14 phenotypes on 13 270 chromosomes $\left(P<1.7 \times 10^{-6}\right.$, the Bonferroni-corrected significance threshold, 271 Supplementary Table 8). Of which, 9 SVs were in the introns, and the remaining 16 272 SVs were in the intergenic regions. One example is that a $114 \mathrm{bp}$ DEL with MAF of 2730.10 on Chromosome 5 was significantly associated with urinary crystal (XTAL). This 274 DEL located in the intron of SLC9A3 (sodium/hydrogen exchanger, isoform 3), that 275 was previously found to be associated with ammonia metabolism which regulated 276 acidic or alkaline in urine $^{35}$ and thus affected the calcium oxalate $(\mathrm{CaOx})$ 277 crystallization $^{36}$.

\section{Population evolution of SVs}

279 Previous population genetics studies have shown the genetic differences between 280 northern and southern Chinese using SNP arrays and NGS-based WGS ${ }^{11,37}$. We herein 281 explored the population genetic properties of SVs between these two regional groups. 282 Principal component analysis (PCA) showed the clear genetic diversity between the 283 two groups, revealing that population structures were consistent with self-reported 284 ancestry (Fig. 5a). We further estimated the differentiation between them. The average 285 value of the fixation index $\left(F_{\mathrm{st}}\right)$ was 0.0032 , which was slightly higher than the value 286 estimated by SNVs $\left(F_{\text {st }}=0.0015\right)$ in a previous study ${ }^{11}$, implying a higher divergence 287 between the two sub-populations in this study. We observed 15 significant signals $\left(F_{\mathrm{st}}>\right.$ 288 0.066, Supplementary Table 9) across the genome, which were clustered into eight 289 peaks on Chromosomes 1, 2, 3, 6, 10, 12, 14 and 19 (Fig. 5b). Among the 15 signals, 290 four SVs were located within four genes, respectively, namely $H C G 4 B, I G H G 3, M U C 4$ 291 and SLC1A7 (Fig. 5c and Supplementary Table 9). The top two $F_{\text {st }}$ clusters were 292 located in the major histocompatibility complex (MHC) region at Chromosome 6 and 
293 in the immunoglobulin heavy (IGH) cluster locus at Chromosome 14. In the MHC

294 region, significantly differentiated SVs located in the exons of $H C G 4 B$ (HLA complex 295 group 4B) and intergenic regions of $H L A-K$ and $H L A-U$ while MHC was a known site 296 of extreme genetic diversity across humanity and was reported to be under selection in 297 East Asian population ${ }^{38}$. (Fig. 5c and Supplementary Table 9). It is also notable that 298 although there were seven SVs were significantly differentiated on the IGH cluster loci, 299 no haplotype block was observed (Fig. 5d), which illustrated the genetic diversity 300 among individuals, suggesting that accumulation and combination of different 301 genotypes of IGH genes might be associated with the immunity adaption to diverse 302 environments. In addition, a 477 bp DEL was observed in intron of SLC1A7, which is 303 a solute carrier family member and has L-glutamate transmembrane transporter activity 304 and was previously reported to be the immune-associated prognosis signature for 305 hepatocellular carcinoma ${ }^{39}$. The differentiation of SVs in the immunity associated 306 regions might be due to genetic drift and long-term expose to diverse environments for 307 sub-populations of Chinese.

\section{Discussion}

309 This study presented one of the largest LRS-based genomic datasets to date. We 310 generated reliable reference sets for SVs and identified a mean of 18,489 high311 confidence SVs per Chinese genome that affected $23.0 \mathrm{Mb}$ of the genome. Our SV 312 datasets was much larger than the ones from SRS-based studies, where an average of 3134,442 and 8,202 SVs per human genome were detected ${ }^{25,40}$. Out of 132,312 non314 redundant SVs described here, 53.3\% were previously unreported. The high number of 315 novel SVs in our study might be due to: (1) the methodological improvements of 316 detecting SVs using LRS technology; (2) the large number of samples in our study; and 317 (3) the inclusion of Chinese population, which has been lowly represented in previous 318 studies.

319 LRS technology such as ONT with relatively high sequence error rates more likely 320 leads to mis-mapping against the reference genome and may cause unreliable SV 
321 detection. We used multi-algorithm ensemble approach and stringent filtering strategy

322 to improve SV detection. In addition, we performed orthogonal validation using PacBio

323 high-fidelity (HiFi) sequencing and PCR experiments. The overall FDR of all the SVs

324 was around $3.2 \%$ and that of singleton SVs was around $6 \%$. Compared to a prior study

325 where an average number of 22,755 SVs per genome was detected by an SV caller,

326 SMRT-SV ${ }^{8}$, our study detected a lower number of SVs per genome $(18,489)$, mainly

327 because we used multiple SV callers and stringent filtering processes. When we applied

328 one caller, such as Sniffles or NanoVar, to detect the SVs, we could detect an average

329 of 20,186 or 31,252 SVs per sample, respectively. When estimating the relationship

330 between the detected SV number and sequencing depth, we found that the number of

331 SVs just became stable when the depth was more than 15 -fold. Our analysis suggested

332 that our strategy was effective to detect SVs for a population-scale study.

333 Our datasets enable us to explore SVs with a low frequency in the population. We

334 provided several lines of evidence that singleton and rare SVs were more likely to be

335 functional. In particularly, pLoF SVs that altered coding regions and affected clinical

336 phenotypes could be rare or even singleton in the population, such as long DELs

337 covering the whole genes of $H B A 1 / H B A 2$ and $H B B$. Indeed, a recent study also

338 discovered rare LoF variants in 26 genes, derived from whole-exome sequencing, were

339 significantly associated with phenotypes ${ }^{41}$.

340 With the rich phenotypic and clinical measurements, our study presents a key step

341 for establishing a regional reference genome and provides a broad prospect to improve

342 the interpretation of clinical genetics in Chinese population. To date, many diseases,

343 such as rare diseases and neurodevelopment-related diseases, have been validated to be

344 caused by $\mathrm{SVs}^{42,43}$. However, it is still a difficult task to find pathogenic SVs, even

345 though conducting a whole-genome scan using LRS approaches, as the effects of SVs

346 still remain largely unknown, particularly for those in noncoding regions. Our SV

347 dataset constructed from a large Chinese population could help future LRS-based

348 genomic studies to narrow down candidates for pathogenic variants. 
In summary, given that the current human reference genome and population

350 genomics have a substantially large number of uncovered SVs, this study presents an

351 important effort to fill this knowledge gap and provides us the opportunity to detect novel SVs associated with phenotypes, diseases and evolution.

\section{Ethics approval and consent to participate}

354 This research was approved by the Ethics Committee of Zhongshan Ophthalmic Center, 355 Sun Yat-sen University (2019KYPJ111) and West China Hospital, Sichuan University 356 (2018-120). Clinical data and biological specimens were obtained from the individuals 357 with written informed consent.

358 Data availability

359 SVs of 405 individuals have been stored in VCF format and deposited into the Genome

360 Variation Map (GVM) database in Beijing Institute of Genomics (BIG) Data Center 361 (https://bigd.big.ac.cn/gsa/index.jsp). The codes of data analysis and PCR validations 362 are available at https://github.com/xie-lab/PGC. All the data and codes will be publicly 363 available.

\section{Competing interests}

365 The authors declare that they have no competing interests.

\section{Funding}

367 This project was supported by National Key R\&D Program of China 368 (2019YFA0904400, Z.X.), National Natural Science Foundation of China 369 (31829002, Z.X; 81630030 and 81920108018, T.L.); Special Foundation for Brain 370 Research from Science and Technology Program of Guangdong (2018B030334001,

371 T.L.); Key R \& D projects of Science and Technology Department of Sichuan 372 Province (2019YFS0535, 2019YFS0039, T.L.); 1.3.5 Project for disciplines of 373 excellence, West China Hospital of Sichuan University (ZY2016103, ZY2016203 374 and ZYGD20004, T.L.). 


\section{Acknowledgements}

376 Dr. Rong Ju provided helpful suggestion. We thank the support of the Center for

377 Precision Medicine at Sun Yat-sen University. We also thank BioMarker Inc. for 378 providing the ONT sequencing platform and Annoroad Gene Technology company

379 for providing the PacBio sequencing platform.

\section{$380 \quad$ Author contributions}

381 Z.X conceived and supervised the study. ZK.W, Z.X, Tao L and YZ. L designed the 382 study. ZK.W, Tong L, ZH.J and Z.X analyzed the data. LS.Z, CB.X, S.OY and Tao

383 L organized and executed the clinical study. JQ.Y conducted PCR experiments.

384 ZK.W, Tong L and ZH.J prepared the manuscript. ZK.W, Z.X and Tong L wrote the 385 manuscript. All authors read and approved the manuscript.

\section{Reference}

387 1. Alkan, C., Coe, B.P. \& Eichler, E.E. Genome structural variation discovery and genotyping. $388 \quad$ Nat Rev Genet 12, 363-76 (2011).

389 2. Malhotra, D. \& Sebat, J. CNVs: Harbingers of a Rare Variant Revolution in Psychiatric $390 \quad$ Genetics. Ce//148, 1223-1241 (2012).

391 3. Coe, B.P. et al. Neurodevelopmental disease genes implicated by de novo mutation and 392 copy number variation morbidity. Nat Genet $\mathbf{5 1}$, 106-116 (2019).

393 4. Ishiura, H. et al. Expansions of intronic TTTCA and TTTTA repeats in benign adult familial $394 \quad$ myoclonic epilepsy. Nat Genet 50, 581-590 (2018).

395 5. Baca, S.C. et al. Punctuated evolution of prostate cancer genomes. Cel/153, 666-77 (2013).

396 6. Mitsuhashi, S. \& Matsumoto, N. Long-read sequencing for rare human genetic diseases. 397 J Hum Genet 65, 11-19 (2020).

398 7. Aganezov, S. et al. Comprehensive analysis of structural variants in breast cancer genomes 399 using single molecule sequencing. biorxiv (2019).

400 8. Audano, P.A. et al. Characterizing the Major Structural Variant Alleles of the Human $401 \quad$ Genome. Cell176, 663-675 e19 (2019).

402 9. Shi, L. et al. Long-read sequencing and de novo assembly of a Chinese genome. Nat $403 \quad$ Commun 7, 12065 (2016).

404 10. Beyter, D. et al. Long read sequencing of 1,817 Icelanders provides insight into the role 405

406 11. Du, Z. et al. Whole Genome Analyses of Chinese Population and De Novo Assembly of A 407

408 12. Cao, H. et al. De novo assembly of a haplotype-resolved human genome. Nat Biotechnol 409 33, 617-22 (2015). 
410 13. Cai, R., Dong, Y., Fang, M., Guo, C. \& Ma, X. De novo genome assembly of a Han Chinese

411

412

413

414

415

416

417

418

419

420

421

422

423

424

425

426

427

428

429

430

431

432

433

434

435

436

437

438

439

440

441

442

443

444

445

446

447

448

449

450

451

male and genome-wide detection of structural variants using Oxford Nanopore sequencing. Mol Genet Genomics (2020).

14. Himmelbauer, H., Stralis-Pavese, N., Peters, P. \& Dohm, J.C. Benchmarking of long-read correction methods. NAR Genomics and Bioinformatics 2(2020).

15. Sedlazeck, F.J. et al. Accurate detection of complex structural variations using singlemolecule sequencing. Nat Methods (2018).

16. Tham, C.Y. et al. NanoVar: accurate characterization of patients' genomic structural variants using low-depth nanopore sequencing. Genome Bio/21, 56 (2020).

17. Cretu Stancu, M. et al. Mapping and phasing of structural variation in patient genomes using nanopore sequencing. Nat Commun 8, 1326 (2017).

18. Chaisson, M.J. et al. Resolving the complexity of the human genome using singlemolecule sequencing. Nature 517, 608-11 (2015).

19. Wenger, A.M. et al. Accurate circular consensus long-read sequencing improves variant detection and assembly of a human genome. Nat Biotechno/ (2019).

20. Robinson, J.T., Thorvaldsdottir, H., Wenger, A.M., Zehir, A. \& Mesirov, J.P. Variant Review with the Integrative Genomics Viewer. Cancer Res 77, e31-e34 (2017).

21. MacDonald, J.R., Ziman, R., Yuen, R.K., Feuk, L. \& Scherer, S.W. The Database of Genomic Variants: a curated collection of structural variation in the human genome. Nucleic Acids Res 42, D986-92 (2014).

22. Collins, R.L. et al. gnomAD-SV an open resource of structural variation for medical and population genetics. (2019).

23. Almarri, M.A. et al. Population Structure, Stratification, and Introgression of Human Structural Variation. Cell (2020).

24. Sungwon Jeon, Y.B., Yeonsong Choi, Yeonsu Jeon, Seunghoon Kim, Jaeyoung Jang, Jinho Jang, Asta Blazyte, Changjae Kim, Yeonkyung Kim, Jungae Shim, Nayeong Kim, Yeo Jin Kim, Seung Gu Park, Jungeun Kim, Yun Sung Cho, Yeshin Park, Hak-Min Kim, \& ByoungChul Kim, N.-H.P., Eun-Seok Shin, Byung Chul Kim, Dan Bolser, Andrea Manica, Jeremy S. Edwards, George Church, Semin Lee, Jong Bhak. Korean Genome Project: 1094 Korean personal genomes with clinical information. Sci. Adv., eaaz7835 (2020).

25. Collins, R.L. et al. A structural variation reference for medical and population genetics. Nature 581, 444-451 (2020).

26. Buniello, A. et al. The NHGRI-EBI GWAS Catalog of published genome-wide association studies, targeted arrays and summary statistics 2019. Nucleic Acids Res 47, D1005-D1012 (2019).

27. Amberger, J.S., Bocchini, C.A., Scott, A.F. \& Hamosh, A. OMIM.org: leveraging knowledge across phenotype-gene relationships. Nucleic Acids Research 47, D1038-D1043 (2019).

28. Tate, J.G. et al. COSMIC: the Catalogue Of Somatic Mutations In Cancer. Nucleic Acids Res 47, D941-D947 (2019).

29. AbdulAzeez, S. \& Borgio, J.F. In-Silico Computing of the Most Deleterious nsSNPs in HBA1 Gene. PLoS One 11, e0147702 (2016).

30. Ta^nia Carlice-dos-Reis, J.V., Fabiano Cordeiro Moreira, Greice de \& Lemos Cardoso, J.G., 
Sidney Santos, A^ ndrea Ribeiro-dos-Santos. Investigation of mutations in the HBB gene using the 1,000 genomes database. PLOS ONE 12, e0174637 (2017).

454 31. Lin, S., Li, C., Li, C. \& Zhang, X. Growth Hormone Receptor Mutations Related to Individual Dwarfism. Int J Mol Sci 19(2018).

456 32. Blum, W.F. et al. The growth hormone-insulin-like growth factor-I axis in the diagnosis and treatment of growth disorders. Endocr Connect 7, R212-R222 (2018).

33. Waxman, D.J. \& O'Connor, C. Growth hormone regulation of sex-dependent liver gene expression. Mol Endocrino/ 20, 2613-29 (2006).

34. Yakar, S. \& Adamo, M.L. Insulin-like growth factor 1 physiology: lessons from mouse models. Endocrinol Metab Clin North Am 41, 231-47, v (2012).

35. Canales, B.K. et al. Polymorphisms in Renal Ammonia Metabolism Genes Correlate With 24-Hour Urine pH. Kidney Int Rep 2, 1111-1121 (2017).

36. Manissorn, J., Fong-Ngern, K., Peerapen, P. \& Thongboonkerd, V. Systematic evaluation for effects of urine $\mathrm{pH}$ on calcium oxalate crystallization, crystal-cell adhesion and internalization into renal tubular cells. Sci Rep 7, 1798 (2017).

37. Chen, J. et al. Genetic structure of the Han Chinese population revealed by genome-wide SNP variation. Am J Hum Genet 85, 775-85 (2009).

38. Chiang, C.W.K., Mangul, S., Robles, C. \& Sankararaman, S. A Comprehensive Map of Genetic Variation in the World's Largest Ethnic Group-Han Chinese. Mol Biol Evol 35, 2736-2750 (2018).

39. Hu, B., Yang, X.B. \& Sang, X.T. Development and Verification of the Hypoxia-Related and Immune-Associated Prognosis Signature for Hepatocellular Carcinoma. J Hepatocell

Carcinoma 7, 315-330 (2020).

475 40. Abel, H.J. et al. Mapping and characterization of structural variation in 17,795 human genomes. Nature (2020).

477 41. Park, J. et al. Exome-wide evaluation of rare coding variants using electronic health records identifies new gene-phenotype associations. Nat Med 27, 66-72 (2021). newly identified by long-read whole-genome sequencing. J Hum Genet (2019). familial cortical myoclonic tremor with epilepsy type 1. Brain 141, 2280-2288 (2018). 


\section{Figures and Tables}
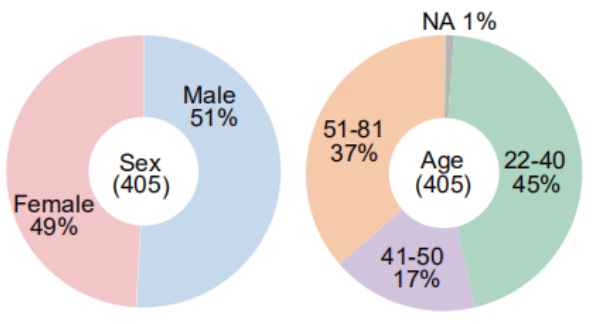

C

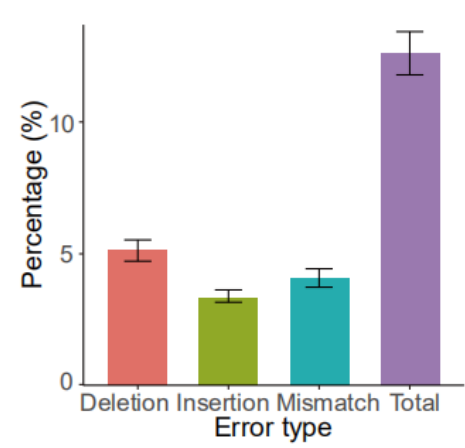

f

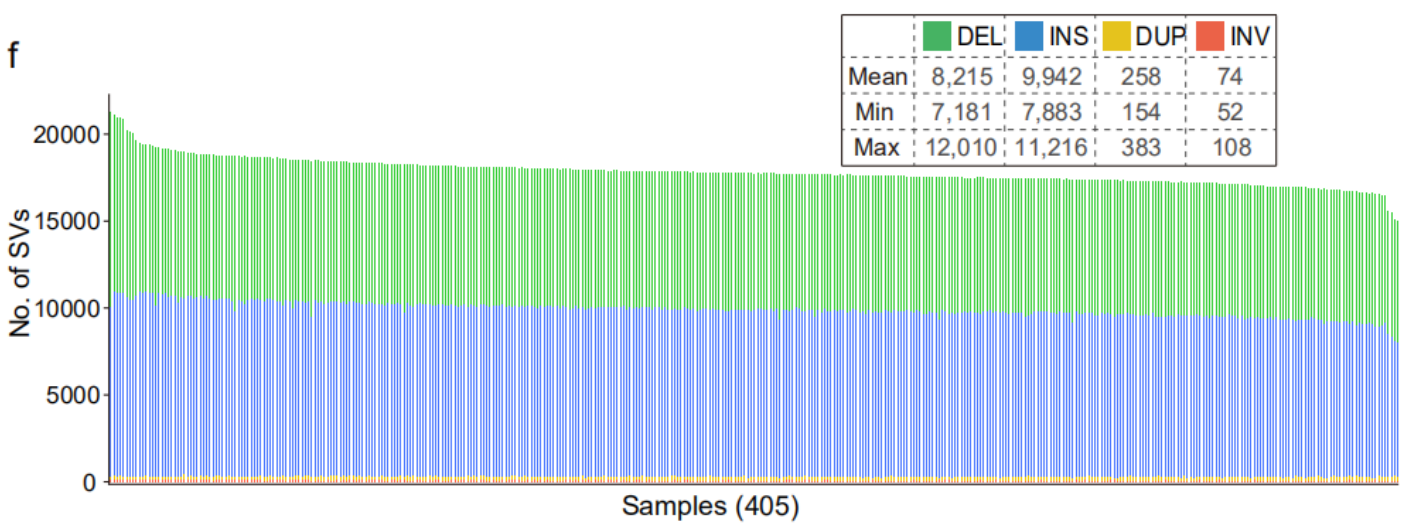

b
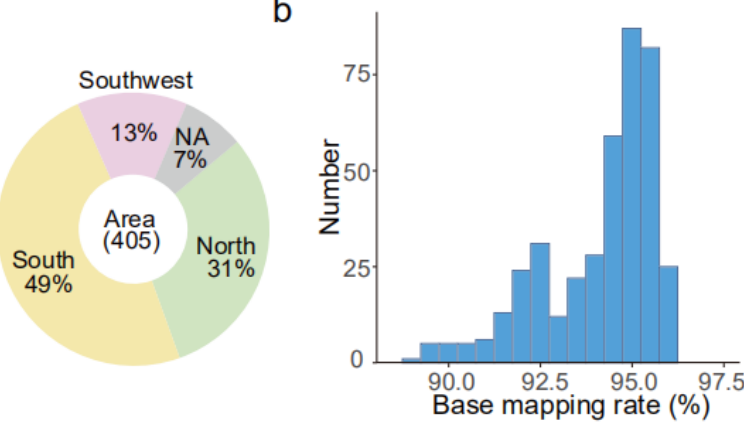

d
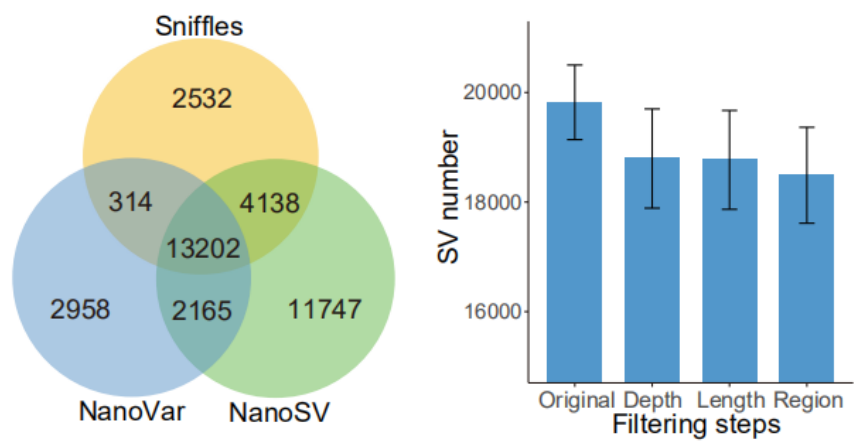

INS: DUP INV

Max

Samples (405)

Figure 1. Overview of samples, datasets and SVs

a, Overview of information of samples in the study, "NA" denotes not available.

b, Distribution of base mapping rate of clean data aligning to the reference genome GRCh38.

c, Error rate for each type, error bars represent standard deviation.

d, Average No. of SVs identified by Sniffles, NanoVar and NanoSV per individual and the overlaps among them.

e, Average No. of SVs after each filtering step, "Original" means unfiltered SVs detected by at least two callers, "Depth", "Length" and "Region" mean SVs after being filtered by supported reads number, extra-long interval and very low complex regions, respectively.

f, No. of SV for each SV type in each individual. 


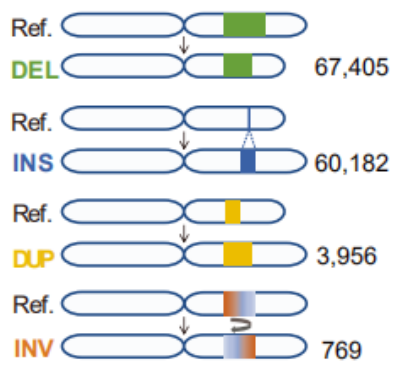

C

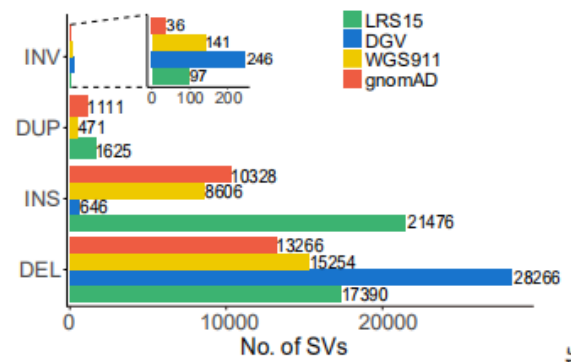

d

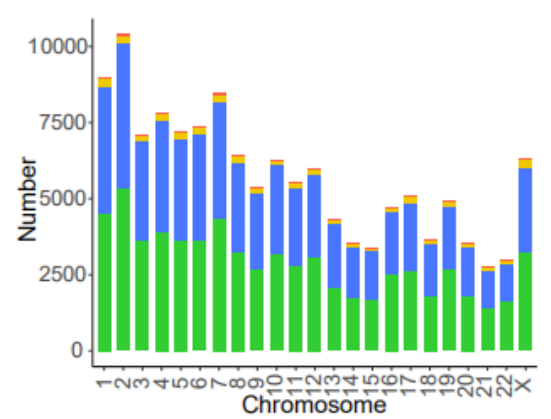

f

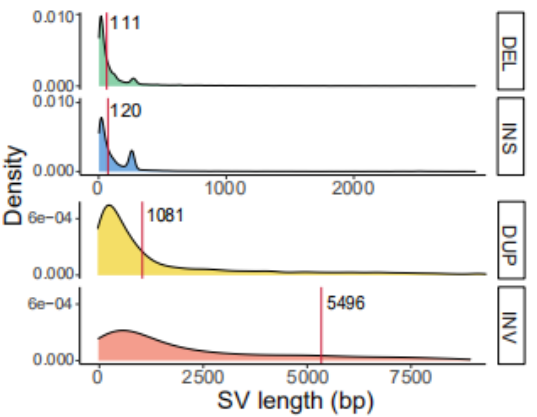

b

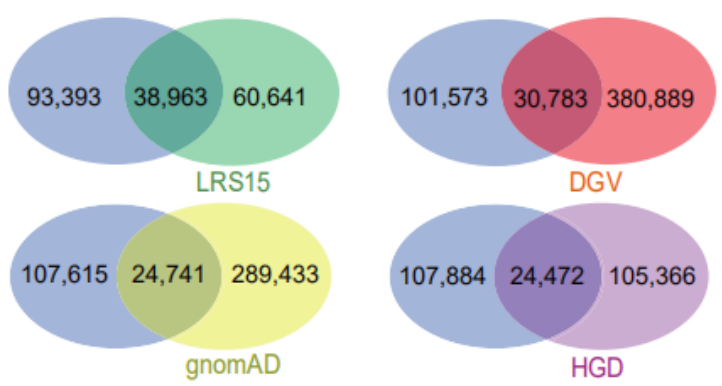

e

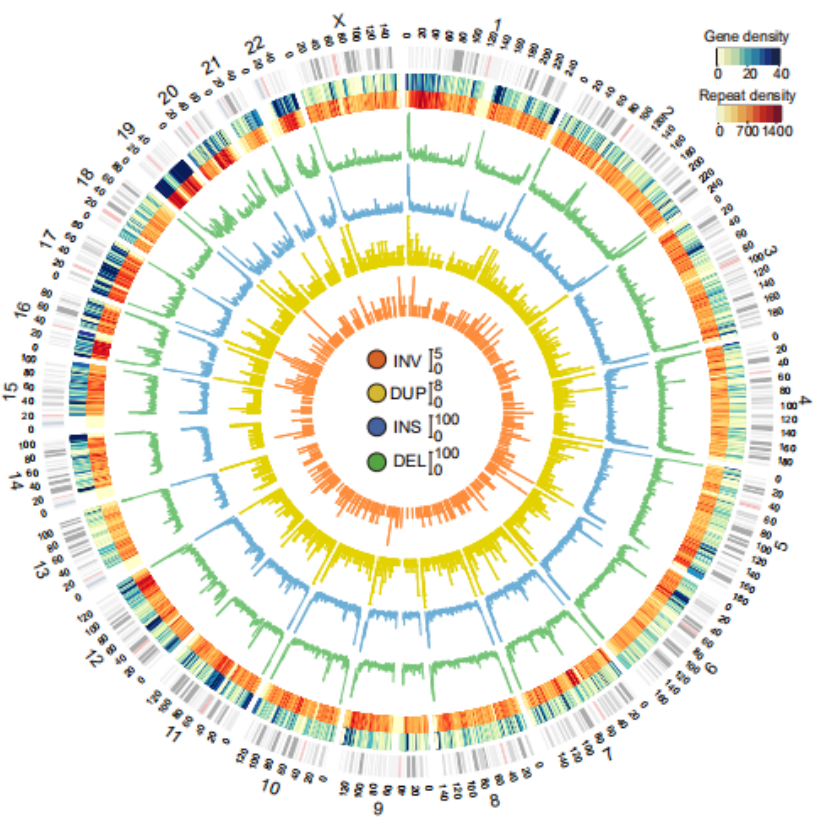

g

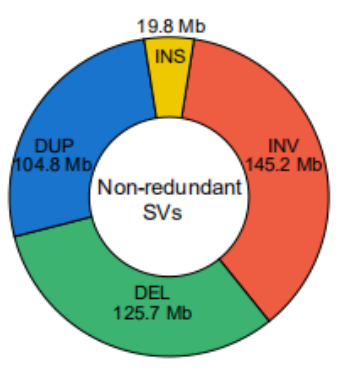

Figure 2. Properties of non-redundant SVs for each SV type

a, No. of non-redundant SVs of all individuals.

b, Overlaps of SVs between our study (blue ellipse) and the previously published datasets.

c, Overlaps of SV number between our study and the previously published datasets for each SV type. The number on the bar graph indicates the actual number of SVs.

d, Total No. of non-redundant SVs for each SV type.

e, No. of genes, repeats and SVs within $500 \mathrm{~kb}$ non-overlapping window across chromosomes. The two outer circles denote the distribution of genes and density of repeats, followed by distributions of DEL (green), INS (blue), DUP (yellow) and INV 
(orange).

f, Length distribution for each SV type. Red line indicates the median length of each SV type.

g, Total length of non-redundant SVs for each SV type across chromosomes.

$\mathbf{h}$, The average No. of individuals for merged non-redundant SVs of each type. 
a

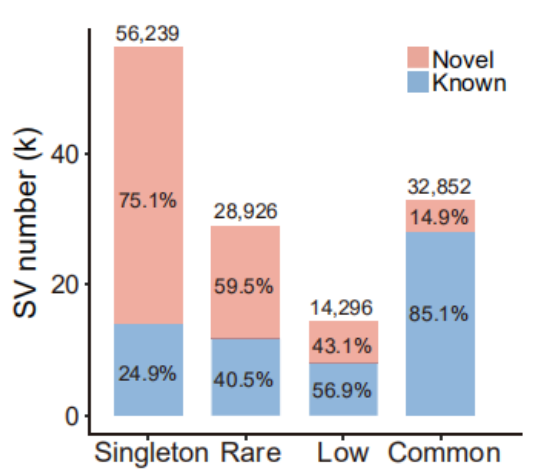

C

\begin{tabular}{|c|c|c|c|}
\hline \multicolumn{2}{|c|}{$\begin{array}{l}\text { predicted Loss- } \\
\text { of-Function }\end{array}$} & \multicolumn{2}{|c|}{$\begin{array}{l}\text { Whole-gene Whole-ger } \\
\text { DUP INV }\end{array}$} \\
\hline No. of SVs & 1,929 & 202 & 146 \\
\hline Median size $(\mathrm{kb})$ & 2.2 & 95.7 & 316.0 \\
\hline SVs per gene & 1.5 & 1.1 & 1.1 \\
\hline Genes per SV & 1.3 & 3.2 & 10.2 \\
\hline SVs per individual & 119.6 & 6.7 & 13.0 \\
\hline
\end{tabular}

b

d
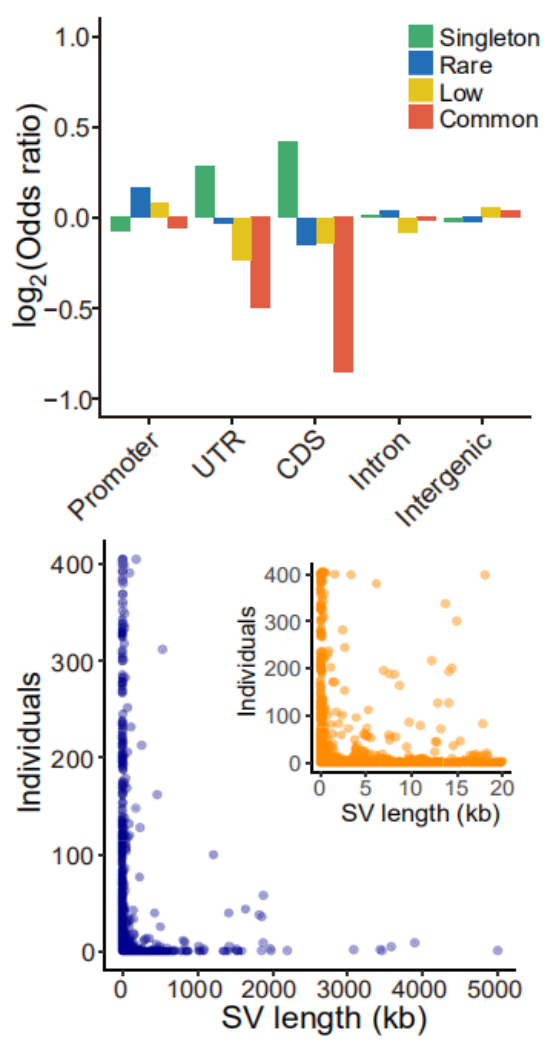

Figure 3. Allele frequency of SVs and functional annotation

a, No. of known and novel SVs for each SV category based on the variant allele frequency $(\mathrm{AF})$ : singleton (allele count $=1$ ), rare (allele count $>1$ and $\mathrm{AF} \leq 0.01$ ), low $(\mathrm{AF}>0.01$ but $\leq 0.05)$ and common $(\mathrm{AF}>0.05)$.

b, Enrichment analysis of genomic location of SVs for each category.

c, Statistics of predicted Loss-of-Function (pLoF) SV, whole-gene DUP (WDUP) and whole-gene INV (WINV).

d, Individual No. versus SV length for pLoF SVs. The blue figure shows SV length from $1-5000 \mathrm{~kb}$ while the orange figure shows that from $0-20 \mathrm{~kb}$. 
a

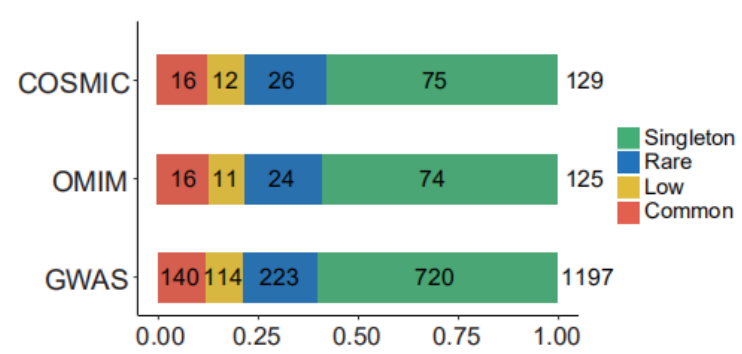

C
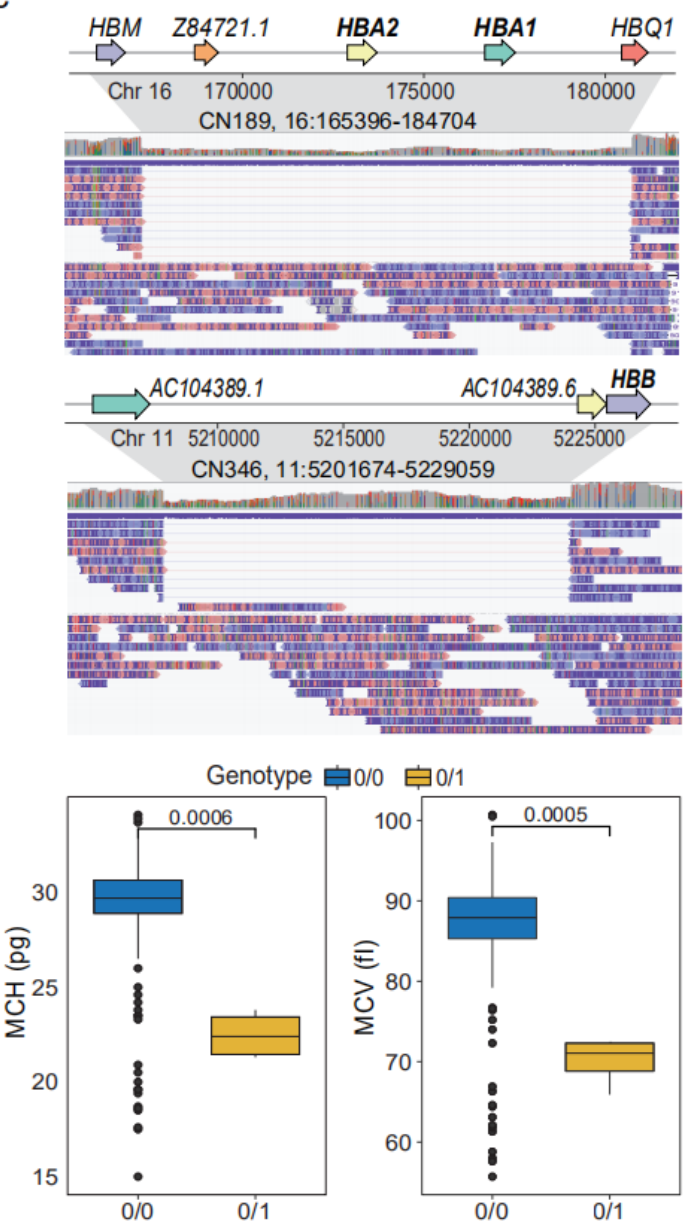

b

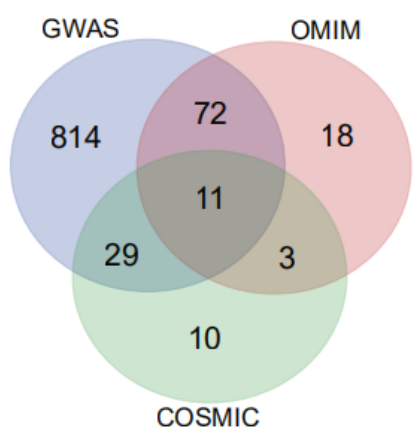

d

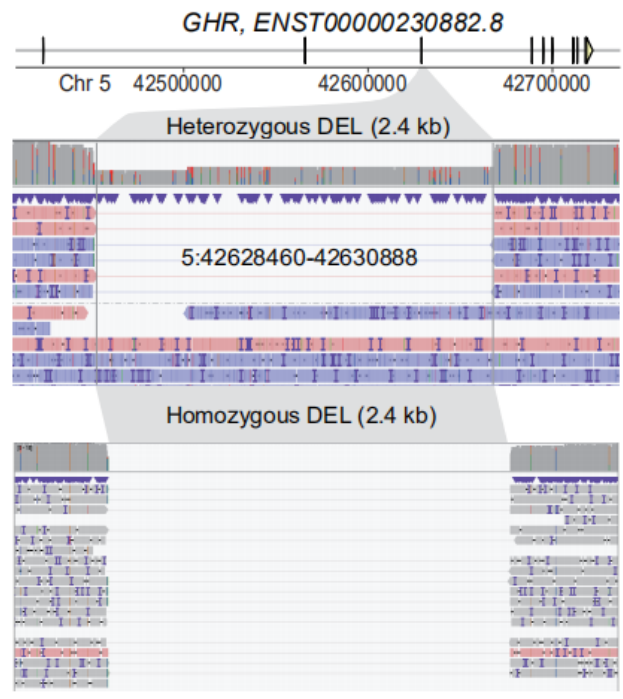

Genotype 追0/0 追0/1 追1/1
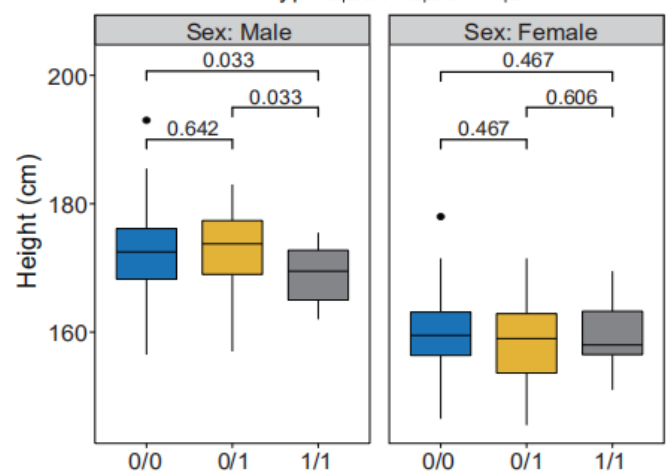

Figure 4. pLoF SVs associated with phenotypes and diseases

a, No. of pLoF SVs with reported genes in GWAS, OMIM and COSMIC for each SV category.

b, No. of genes associated with pLoF SVs.

c, Example of SVs affecting $H B B$ and $H B A 1 / H B A 2$ which are associated with anemia. Up: IGV screenshot of a $19.3 \mathrm{~kb}$ heterozygous DEL covering both $H B A 1$ and $H B A 2$; Middle: IGV screenshot of a $27.4 \mathrm{~kb}$ heterozygous DEL covering $H B B$; Bottom: mean corpuscular hemoglobin $(\mathrm{MCH})$ and mean corpuscular volume (MCV) values of four individuals containing a $19.3 \mathrm{~kb}$ DLE (3 individuals) and a $27.4 \mathrm{~kb}$ DEL (1 individual) and the others.

d, Example of SVs affecting $G H R$ which is associated with height of male adults. Up: IGV screenshot of a $2.4 \mathrm{~kb}$ heterozygous DEL completely covering the third exon of 
GHR; Middle: IGV screenshot of the $2.4 \mathrm{~kb}$ homozygous DEL. The $p$ values were derived from multiple testing correction. For $\mathbf{c}$ and $\mathbf{d}$, " $0 / 0$ ": homozygous allele same as the reference; " $0 / 1 "$ ": heterozygous variant; "1/1": homozygous variant. 
a

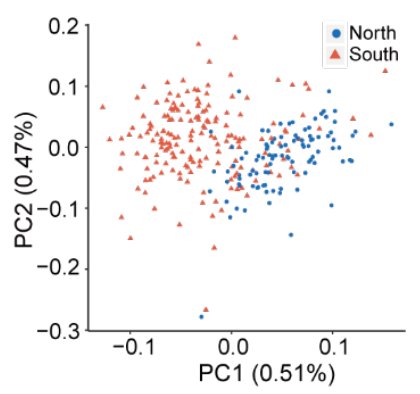

C

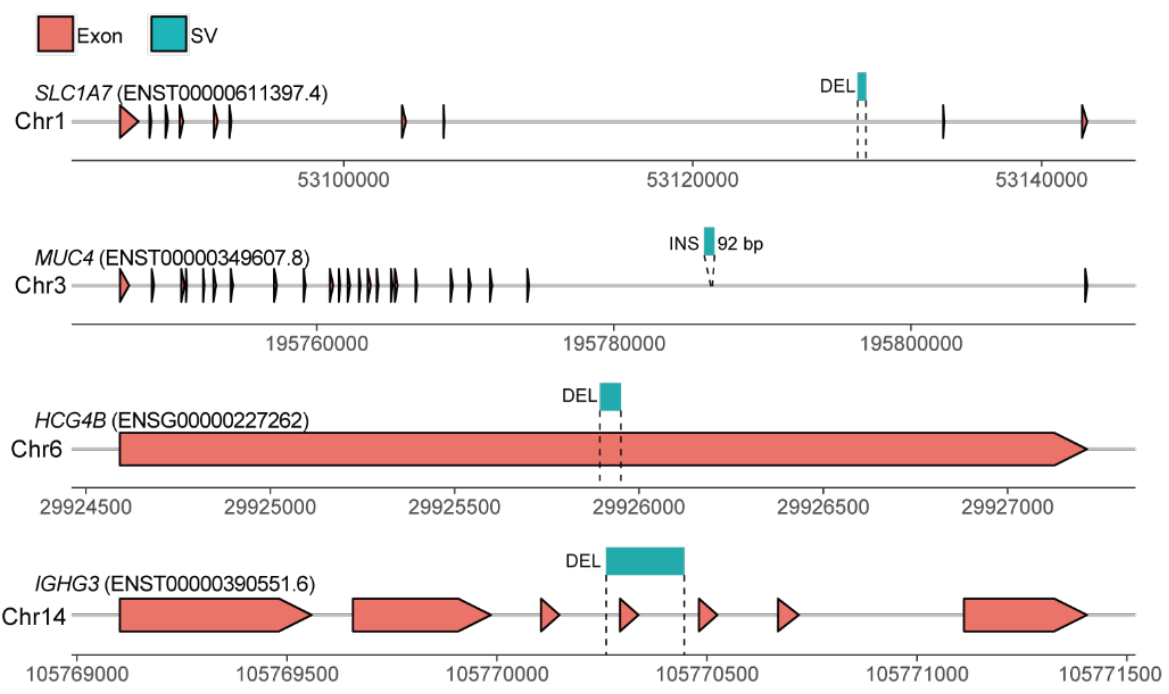

b

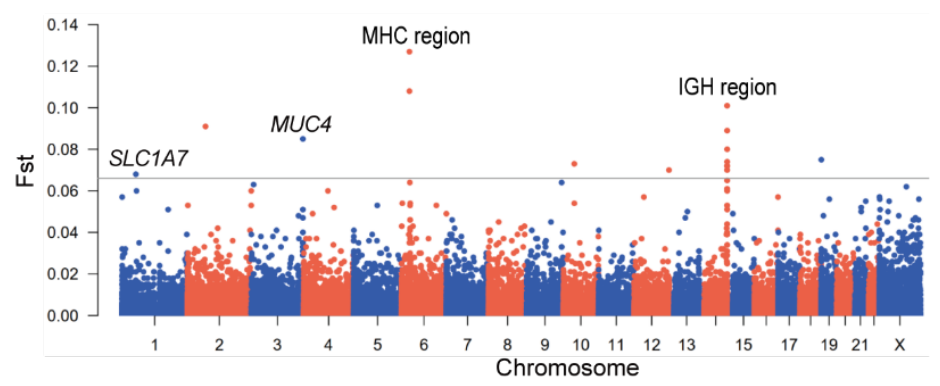

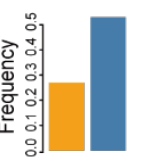
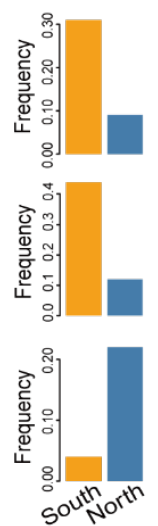

d

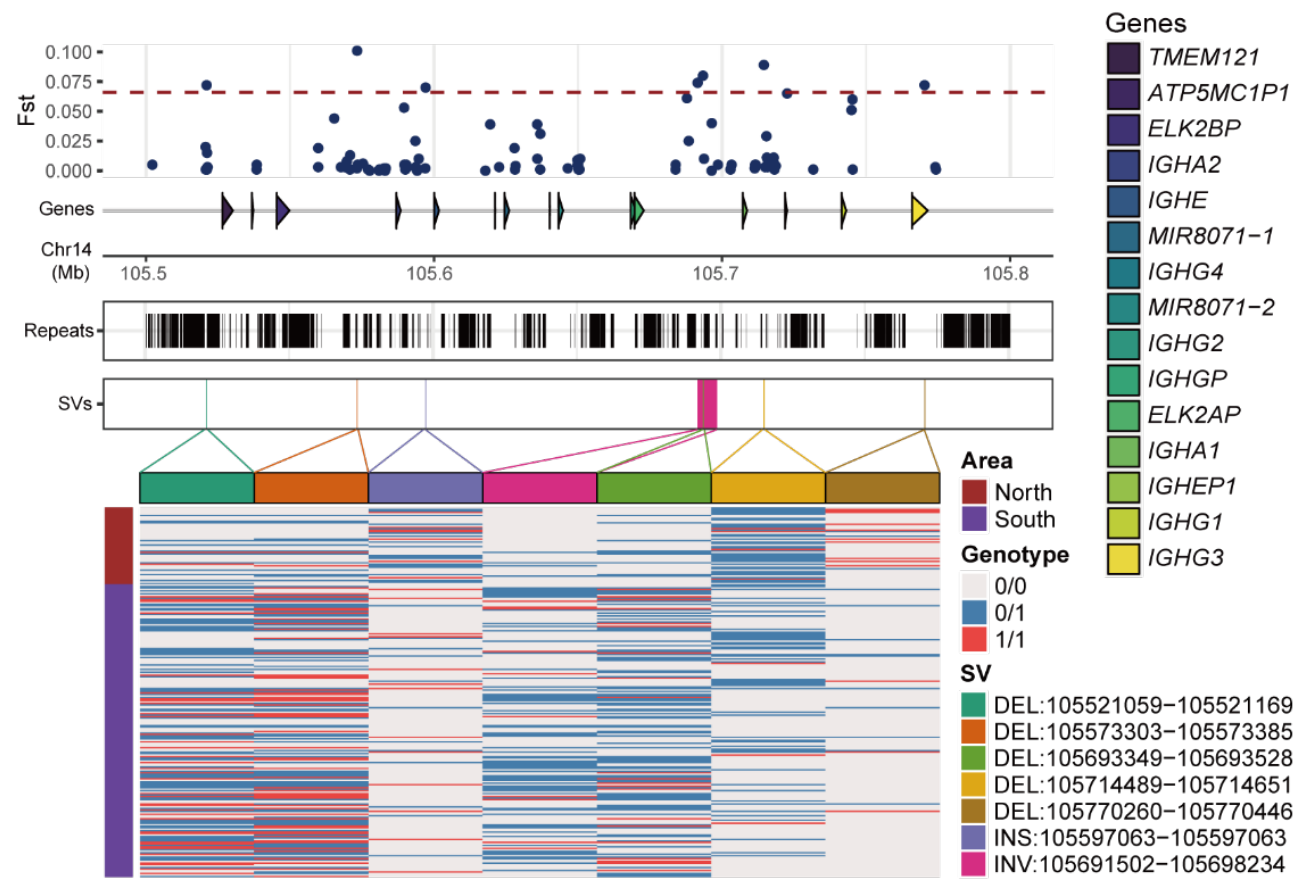

Figure 5. Genetic differentiation of SVs between sub-populations of Chinese

a, PCA of the two sub-populations: northern and southern Chinese. The values in parenthesis indicate the genetic variations explained by first two PCs.

b, $F_{\text {st }}$ value between the two sub-populations. The gray horizontal line indicates the $F_{\text {st }}$ cutoff of $>0.066$ based on permutation. 
c, Four significantly differentiated SVs located in genes. Barplots on the right panel showing the frequency of each SV between northern and southern sub-populations.

d, Differential SVs clumped to IGH region in chromosome 14 and the genotype patterns between the two sub-populations. 
Table 1. Gene Annotation for SVs in Each SV Category

\begin{tabular}{ccccccc}
\hline Category & Promoter (\%) & UTR (\%) & CDS (\%) & Intron (\%) & Intergenic (\%) & All \\
\hline Singleton & $542(1.0)$ & $594(1.1)$ & $1,271(2.3)$ & $21,311(37.9)$ & $32,521(57.8)$ & 56,239 \\
Rare & $329(1.1)$ & $245(0.8)$ & $441(1.5)$ & $11,204(38.7)$ & $16,706(57.8)$ & 28,925 \\
Low & $154(1.1)$ & $105(0.7)$ & $219(1.5)$ & $5,076(35.5)$ & $8,742(61.1)$ & 14,296 \\
Common & $320(1.0)$ & $201(0.6)$ & $303(0.9)$ & $12,208(37.2)$ & $19,820(60.3)$ & 32,852 \\
All & $1,345(1.0)$ & $1,145(0.9)$ & $2,234(1.7)$ & $49,799(37.6)$ & $77,789(58.8)$ & 132,312 \\
\hline
\end{tabular}

Number and percent of SVs affecting the promoter (1 kb upstream of gene), untranslated region (UTR), coding sequence region (CDS) and intron of protein coding gene. SVs that were not intersected with gene were annotated as intergenic region. 


\section{Methods}

\section{Sample information}

A total of 405 individuals were enrolled in this study (206 males and 199 females) with age varying from 22 to 81 years old (Fig. 1a). These individuals came from 18 provinces in China according to their self-reported original province. Northern and southern Chinese were distinguished based on Qinling Mountain-Huaihe River Line. Among them, 329 individuals were recruited at the Health Service Center of Sun Yat-sen University Cancer Center, where 68 clinical phenotypes from 327 individuals were collected. Additional 76 individuals from the West China Hospital of Sichuan University were included in this study. Written informed consent was obtained for all the individuals.

\section{Phenotype collection}

Anthropometric measurements, including height, weight and body mass index (BMI), were obtained from an automatic electronic meter (SECA GM-1000, Seoul, Korea). Blood tests were processed by a hematology automated analyzer (SYSMEX XE-2100, Kobe, Japan). Urine tests were determined using an automated urine chemistry analyzer (ARKRAY4030, Tokyo, Japan) and a urinary tract infection analyzer (SYSMEX UF-1000i, Kobe, Japan). Biochemical detection was performed by an automatic modular analyzer (Cobas C701, Basel, Switzerland). Tumor markers such as alpha fetoprotein (AFP) and carcinoembryonic antigen (CEA) were measured by an immunology modular analyzer (Cobas 8000 e602, Basel, Switzerland).

\section{Library construction and long-read sequencing}

High molecular weight genomic DNA of each individual was extracted from peripheral blood leukocytes using HiPure Tissue \& Blood DNA Kit (D3018-03, Angen). For the Nanopore sequencing, DNA repair, end repair and adapter ligation were conducted during library preparation, and $2 \mu \mathrm{g}$ DNA was Fragmented by g-TUBE (Covaris). DNA repair was performed using NEBNext FFPE DNA Repair Mix (M6630L, NEB). End repair was performed using NEBNext Ultra II End Repair/dA-Tailing Module (E7546L, NEB). Adapter ligation was performed using NEBNext Blunt/TA Ligase Master Mix (M0367L NEB) and Ligation Sequencing Kit 1D (SQK-LSK109, Oxford Nanopore Technologies). DNA was purified between each step using Agencourt AMPure XP beads (A63882, Beckman Coulter). DNA was quantified via a Qubit Fluorometer 2.0 (ThermoFisher Scientific, Waltham, MA). We carried out long-read sequencing using a PromethION sequencer and 1D flow cell with protein pore R9.4.1 1D chemistry according to the manufacturer's instructions. Reads were base-called in 
batches by guppy v3.2.8 using the default parameters during sequencing.

For the PacBio sequencing, the integrity of the DNA was determined with the Agilent 4200 Bioanalyzer (Agilent Technologies, Palo Alto, California). Eight micrograms of genomic DNA were sheared using g-Tubes (Covaris), and concentrated with AMPure PB magnetic beads. Each SMRT bell library was constructed using the Pacific Biosciences SMRTbell template prep kit 1.0. The constructed library was size-selected by Sage ELF for molecules $11 \sim 15 \mathrm{~kb}$, followed by primer annealing and the binding of SMRT bell templates to polymerases with the DNA Polymerase Binding Kit (Pacific Bioscience). Sequencing was carried out on the Pacific Bioscience Sequel II platform for 30 hours.

\section{Read quality control and mapping}

We detected lower quality bases at two ends of reads by NanoQC ${ }^{1} \mathrm{v} 0.8 .1$ and trimmed 30 bases of start and 20 bases of end for each raw read using NanoFilt ${ }^{1}$ v2.2.0 due to their lower quality. We kept the reads with length longer than $500 \mathrm{bp}$ and mean quality higher than seven for downstream analysis. The statistics of length and quality value of clean reads was performed using NanoPlot ${ }^{1}$ v1.20.0. The clean reads were then aligned to the primary assembly of human reference genome GRCh38 using minimap2 $2^{2}$ v2.15-r905 with the recommended option for ONT reads (-x map-ont) and the additional parameters "--MD -a". Aligned files with SAM format were converted to BAM format and then sorted using SAMtools ${ }^{3}$ v1.9. Summary of aligned information for BAM file was conducted by command "stat" of SAMtools and region depth of aligned file was estimated by mosdepth ${ }^{4} \mathrm{v} 0.2 .5$. The read base mapping rate and sequencing error rate were estimated with the method previously described ${ }^{5}$. Specifically, we aligned the cleaned reads to GRCh38 and estimated the rates of substitutions, insertions and deletions based on the mapping result, excluding secondary alignments and the soft-clipped sequences.

\section{Detection of high-confidence SVs}

To obtain high-confidence SVs, we employed multiple tools to call SVs and conducted a series of filtering steps (Supplementary Fig. 2a). Sniffles ${ }^{6}$ v1.0.10, NanoVar ${ }^{7}$ v1.3.6 and NanoSV v1.2.4, which were SV callers specifically designed for long-read, were used to detect SVs. Sniffles was used with parameters "--min_support 2 --min_length 50 --num_reads_report -1 -min_seq_size 500 --genotype --report_BND --report_seq". We set the following parameters for NanoSV: “--data_type ont --mincov 2 --minlen 50". NanoVar had been run with the default parameters. 
We merged the SV call sets of each individual derived from the above three SV callers for each type SV. We applied the Cluster Affinity Search Technique algorithm (CAST) ${ }^{5,9}$ to merge SVs independently for each SV type based on the variant position and length ${ }^{5}$. In order to facilitate the implementation of this algorithm for INSs, the end coordinate of INS was set as the sum of start coordinate and the SV length. First, we segregated all discovered SVs into nonoverlapping groups. For each group, we represented SVs as nodes in a graph and drew an edge between two SVs if they had a minimum mutual overlap of at least $50 \%$ of the length. The SV merging can be modeled by a corrupted clique graph. Consequently, the merged SVs detected by at least two callers were extracted. As suggested by benchmark analysis of LRS callers in our study (Nicolas Dierckxsens et al., unpublished), Sniffles showed the most balanced performance following by NanoVar and NanoSV for ONT reads. Therefore, we prioritized the results of Sniffles, followed by NanoVar. To obtain high-quality SVs, we then conducted three steps to filter out lower quality SVs (Supplementary Fig. 2a). First, we extracted SVs that were supported with at least three reads. In addition, INSs and DELs with length larger than 2 $\mathrm{Mb}$ were discarded. While INVs and DUPs larger than $5 \mathrm{Mb}$ were discarded. Furthermore, sites in the centromere region with length of $61.9 \mathrm{Mb}$ in 22 autosomes and the $\mathrm{X}$ chromosome were removed from further analysis. We identified and discarded SVs intersected with gap regions (marked as "N") and high depth regions ( $\geq 500 \times$, estimated by mosdepth ${ }^{4} \mathrm{v} 0.2 .5$ ) with BEDTools ${ }^{10} \mathrm{v} 2.27 .1$. The genomic position of gap and centromere regions were downloaded via the UCSC Tablebrower ${ }^{11}$.

To discern the relationship between the discovery rate of SVs and sequencing depth using different SV callers, we applied the above strategy to the Nanopore (ONT) reads of HG002 from Genome in a Bottle (GIAB). First, we got the sparse sequences with different depths ranging from $8 \times$ to $40 \times$ by randomly selecting reads from the deep sequencing clean data of HG002. Then the mapping tool and SV callers coupled with the same parameters in this study were used to detected SVs for each dataset. The method "Combine" means the SV shared by at least two of three callers (Supplementary Fig. 2b). The ratio threshold for SV supported reads was 0.2 , which was equal to 3 supported reads for 15 -fold data. We found that a single caller was not sufficient to obtain high-confidence SVs, especially for depth lower than $10 \times$. The results combined from callers with higher sensitivity (NanoSV) and precision (NanoVar), and balanced performance (Sniffles) can be used for detecting high-confidence SVs. At the same time, our stringent strategy might discard some true SVs, which contributed to the smaller number of detected SVs compared with previously study ${ }^{12}$. In addition, the SV number became stable when the sequencing depth was more than $15 \times$. For Combine method, 19,070 SVs were 
detected at depth of $15 \times$, which was $93.6 \%$ of the total number $(20,378)$ at depth of $40 \times$ (Supplementary Fig. 2b), indicating that the median depth $(15 \times)$ sequencing data was a costeffective method for genetic research at a population-scale.

\section{Non-redundant SVs and genotypes in the population}

A large proportion of SVs were carried by multiple individuals because of their genetic similarity within population. To remove redundancies, we merged SVs of all the individuals using CAST algorithm. Any region that frequently occurred across the population was selected to represent the non-redundant SVs. SVs were genotyped based on the variant allele balance (VAB). The genotype of individual was assigned as " $0 / 0$ " if $\mathrm{VAB} \leq 0.2$, and genotype was " $0 / 1$ " and " $1 / 1$ " for $0.2<\mathrm{VAB} \leq 0.8$ and $\mathrm{VAB}>0.8$, respectively ${ }^{13}$. The threshold was same to prior study by Pedersen et al. ${ }^{13}$ and similar to that of vg toolkit ( 0.14 for default $)$ when applying graph genotyping ${ }^{14}$.

After genotyping, the merged SVs were classified with four categories based on the variant allele frequency $(\mathrm{AF})$ : singleton (allele count $=1$ ), rare (allele count $>1$ and $\mathrm{AF} \leq 0.01$ ), low $(0.01<\mathrm{AF} \leq 0.05)$ and common $(\mathrm{AF}>0.05)$. To estimate the relationship between nonredundant SV number for different categories and sample size, we merged SVs randomly sampled from 100 to 405 samples while setting step size as 3, and repeated four times for each step. Then we calculated SV number for each category and regarded the average of four times as the estimate value of each step. We observed that the number of common SVs in population was relatively stable (Supplementary Fig. 6). As samples increased, the number of low SVs decreased and rare SVs increased, and the steps appeared when the sample number was a multiply of 50 due to the same integer threshold in this period. However, the total number of low and rare SVs increased with similar trend of singletons.

\section{False discovery rate (FDR) of detected SVs}

In order to estimate the false discovery rate (FDR) for the detected SVs, we applied the strategy used in this study to the published dataset comprising of a parent-offspring trio with ONT and PacBio high-fidelity (HiFi) reads (accuracy $>99 \%$ ). The depths of PacBio HiFi reads for HG002, HG003 and HG004 were 19.5×, 21.9×, and 21.6×, respectively. Simultaneously, we randomly selected $15.1 \times$ depth sequences from cleaned ONT reads for HG002 (child). After detecting SVs for each dataset via the same strategy as stated before, we compared SVs from HG002 with ONT reads to those of the trio with HiFi reads. For the 18,737 SVs detected by 
ONT reads, there were $1,165(6.2 \%)$ SVs not detected by HiFi reads of the trio. After manual investigation of IGV snapshot of HG002 with ONT reads and the corresponding PacBio HiFi reads, we finally found 608 false positive SVs in HG002 with ONT reads, and the FDR of SV detection was 3.2\%. Among them, false positives for DEL, INS, DUP and INV were 459 $(5.4 \%), 133(1.3 \%), 11(6.3 \%)$ and $4(5.3 \%)$, respectively.

Singletons are known to have a higher error rate compared with the other categories because they existed in only one sample. To further orthogonally validate the accuracy of singletons uncovered in this study, we sequenced PacBio high-fidelity (HiFi) reads for CN365 (10.5×), CN366 (7.7×), CN371 (7.8×) and CN372 (11.4×) and then detected SVs applying above method. Among 510 singletons discovered by ONT reads for these samples, $32 \mathrm{SVs}$ were false positive based on the validation of PacBio HiFi reads and manual curation, with an FDR of $6.3 \%$.

Besides the orthogonal validation for singletons using PacBio HiFi data, we further validated singletons using PCR experiments. We randomly selected singletons from 20 samples with SV lengths ranging from 60 to 810 bp (average of 293 bp) (Supplementary Table 6) and designed the primers using BatchPrimer $3^{15}$ to amply the SV fragments. We conducted each PCR for positive sample, followed by negative sample and purified water without DNA, which were consider as negative control. Totally, we designed primers for randomly selected 154 DELs and INSs. Among them, amplified lengths for 145 (94.2\%) primers were consistent with the targets, and nine primers failed to amplify target fragments (https://github.com/xielab/PGC/tree/master/data/PCR). The estimated FDR of singletons was 5.8\% (Supplementary Table 6).

\section{SV density of Meta-chromosome}

To compute the density of SVs of chromosomes, we normalized the lengths of all 22 autosomes and $\mathrm{X}$ chromosome. First, we split the chromosomes into p-arm and q-arm. The value of 0 to 1 corresponded to the telomere to centromere of $\mathrm{p}$-arm, and the value of 1 to 2 corresponded to the centromere to telomere of q-arm. For each arm, we set a window of $100 \mathrm{~kb}$ and then calculated the SV number in each overlapping window. We normalized value of each window based on the positions relative to total length of each arm.

\section{Comparison of non-redundant SVs to the published datasets}

To assess the known and novel SVs for our non-redundant SV call set, we compared it to some published datasets, including LRS study of 15 human genomes (LRS15) ${ }^{12}$, Database of 
Genomic Variants (DGV, release 2-25-2020) ${ }^{16}$, Genome Aggregation Database (gnomAD v2.1 $)^{17}$ and Human Genome Diversity panel (HGD) ${ }^{18}$. We extracted the position relative to GRCh38 and length information for each SV. The hg38 coordinates of gnomAD was converted by LifeOver (https://genome-store.ucsc.edu//) ${ }^{19}$ based on the original hg37 version. Copy number variation (CNV) with copy gain and copy loss were regarded as DUP and DEL, respectively. Additionally, the mobile element insertion (MEI) in those datasets were considered as INS. The end position of INS was defined as the sum of original end and the length of INS when comparing INSs between different datasets. We excluded INSs whose insertion length was not available because both SV length and position information should be taken into account. To compare with our dataset, we conducted algorithm CAST to independently remove redundant SVs for each downloaded dataset as described in SVs merging. Intersected regions for each SV type between our study and the published datasets were conducted using BEDTools, and SVs were considered as overlapped if the reciprocal overlap was larger than $50 \%$.

\section{Repeat analysis of SV sequence}

In order to better evaluate the pattern of repeat sequences for SVs, we selected the sequence of the individual with longest SV length in each merged SV. Consequently, we successfully obtained 55,476, 42,912, 3,956 and 770 sequences for DEL, INS, DUP and INV, respectively (Supplementary Table 3). In aggregate, 103,114 (77.9\% of total SVs) sequences were used for downstream analysis of repeat pattern. The repeat sequences were searched by RepeatMasker v4.0.9 (http://www.repeatmasker.org) based on databases of Dfam ${ }^{20} \mathrm{v} 3.0$ and RepBase $^{21}$ (release 10-26-2018) with command "RepeatMasker -species human -pa threads gff -dir output sv_seq.fa" and Tandem Repeat Finder (TRF) ${ }^{22}$ v4.09 with command "trf 277 $801050500-\mathrm{f}-\mathrm{d}-\mathrm{m}$ ”. Each SV was classified into the repeat family if it was occupied by more than half of the SV length. For tandem repeats, the repeat unit length $\geq 7 \mathrm{bp}$ were annotated as variable number of tandem repeats (VNTR). The VNTR regions for genome reference GRCh38 were downloaded via UCSC Table brower.

\section{Gene features of SVs}

We annotated detected SVs based on the known protein-coding gene annotation file (gtf) corresponding GRCh38 from Ensembl. We detected the intersection of SVs using BEDTools. Promoter was defined as the $1 \mathrm{~kb}$ region directly preceding the transcription start site of gene. We predicted Loss-of-Function (pLoF) SVs as follows: (1) DEL: overlap with at least one CDS; 
(2) INS: insertion directly into any CDS; (3) DUP and INV: partially overlap with at least one CDS. In addition, INV and DUP were generally long, we hence defined DUP and INV that covered the whole-gene as WDUP and WINV, respectively ${ }^{23}$. We did not consider WDUP and WINV as gene-disruptive SVs, although we cannot rule out the possibility that they might enhance or regulate gene expression via duplication or cis-action. In addition, we labeled SVs as UTR-disruptive if at least one breakpoint was in 5' or 3' UTR and this SV was not intersected with CDS. Then we labeled SVs as promoter-disruptive if at least one breakpoint was in promoter of a gene and this SV was not intersected with CDS and UTR. We labeled SVs as intron-disruptive if both breakpoints were in same gene and this SV did not meet any of the above criteria. Ultimately, the remaining SVs that were not intersected with any protein-coding gene region (including promoter) was labeled as intergenic.

\section{Enrichment analysis of pLoF SVs and associated genes}

For enrichment analysis of each gene feature annotation of SVs, the expected value was defined as the SV number in gene feature divided by the total number of SVs in population, and the SV number in certain category of this feature divided by the total number of SVs in this category was considered as observed value. The Fisher's exact test was conducted in $\mathrm{R}^{24}$ v3.5.3 (http://www.R-project.org/). To assess functions and associated pathways of pLoF SVs, we performed enrichment analysis using GSEApy v0.9.16 (https://github.com/zqfang/GSEApy). The annotation files, including GO_Molecular_Function_2018, KEGG_2019_Human, GWAS_Catalog_2019, OMIM_Expanded, were downloaded from the Enrichr ${ }^{25}$ website (https://amp.pharm.mssm.edu/Enrichr). The p value was calculated with Fisher's exact test, and multiple testing of $\mathrm{p}$ values were corrected by Benjamini-Hochberg method ${ }^{26}$.

\section{Population stratification and differentiation analysis}

To assess the population stratification between northern or southern Chinese sub-populations, we performed principal component analysis (PCA) using EIGENSOFT ${ }^{27}$ v7.2.1. Previous study indicated that distinctly defined population structure was uncovered by DELs in comparison with other type SVs ${ }^{18}$, such as INSs or DUPs. Therefore, 56,544 DELs was used to estimate population stratification after filtering out DELs uniquely existing in southwest Chinese in this study. $F_{\text {st }}$ between northern and southern Chinese sub-populations was estimated based on SVs of the individuals with self-reported ancestry information. The Hudson's estimator $F_{\mathrm{st}}\left(\left(\mathrm{H}_{\mathrm{t}}-\mathrm{H}_{\mathrm{s}}\right) / \mathrm{H}_{\mathrm{t}}\right)^{28}$ between northern and southern Chinese was calculated. $\mathrm{H}_{\mathrm{t}}$ indicates heterozygosity between subgroups, $\mathrm{H}_{\mathrm{s}}$ indicates average heterozygosity within 
subgroups. Here $\mathrm{H}_{\mathrm{t}}=\left(\mathrm{p}_{1}+\mathrm{p}_{2}\right) *\left(2-\mathrm{p}_{1}-\mathrm{p}_{2}\right) / 2, \mathrm{H}_{\mathrm{s}}=\mathrm{p}_{1} *\left(1-\mathrm{p}_{1}\right)+\mathrm{p}_{2} *\left(1-\mathrm{p}_{2}\right), \mathrm{p}_{1}$ and $\mathrm{p}_{2}$ indicate the allele frequency in two sub-groups, respectively. We used a permutation approach to estimate the significant threshold of observed $F_{\text {st }}$ values. For northern Chinese, 52 individuals from provinces with high-latitude (Jilin, Liaoning, Heilongjiang, Neimenggu, Ningxia and Qinghai) were selected. To determine the significant threshold of $F_{\text {st }}$, individuals (198 southern Chinese and 52 northern Chinese) from the two groups were randomly split into two sets of the original size for 1,000 times. The max $F_{\text {st }}$ values across all permutations were recorded and finally arranged in descending order. The five percentile $F_{\text {st }}$ value $(0.066)$ of ranked values was the empirical genome-wide significance threshold for the overall significance level of $\alpha=0.05^{29}$. Manhattan plot was performed using modified script based on qqman (https://github.com/ stephenturner/qqman).

\section{Genotype-phenotype association analysis}

The 29,510 SVs with minor allele frequency (MAF) larger than 0.05 in 327 individuals with clinical phenotypes were used for the analysis. The genome-wide association study (GWAS) was performed using PLINK ${ }^{30}$ v1.90b4 with linear regression under an additive genetic model for the quantitative traits, and age, sex, body mass index (BMI), and the first two principal components were included as covariates. When applying BMI GWAS, BMI itself was excluded from covariates. The association test for case-control was conducted using logistic regression module. We set the genome-wide significance threshold as $5 \times 10^{-8}$, and the significance threshold was set to be $1.7 \times 10^{-6}$ through Bonferroni correction $(0.05 / 29,510)^{31}$.

\section{Visualization of SVs with long-reads}

Visualization of detected SVs was performed using Integrative Genomics Viewer (IGV) ${ }^{32}$ v2.8.6 which was specially updated for viewing variants of long-read. For target SVs, parameter "Link supplementary alignments" was selected to clearly identify heterozygous SVs based on the split reads. For INVs, the linked long reads with different colors (red and blue) indicated different strands when aligning to reference genome.

\section{Statistical analysis}

The statistical tests used were described throughout the article and in the figures. The onetailed Student's $t$ test was performed to compare the clinical phenotype level between different genotypes of genes. We performed FDR correction (https://www.sdmproject.com/ utilities/?show=FDR) for multiple comparisons. The enrichment analysis of singletons for 
bioRxiv preprint doi: https://doi.org/10.1101/2021.02 09.430378; this version posted February 10, 2021. The copyright holder for this preprint (which was not certified by peer review) is the author/funder, who has granted bioRxiv a license to display the preprint in perpetuity. It is made available under aCC-BY-NC-ND 4.0 International license.

different gene location was conducted by Fisher's exact test. Benjamini-Hochberg corrected of $P$ value was used for multiple test analysis. Pearson correlation coefficient was estimated for correlation analysis. All statistical tests were performed in $\mathrm{R}^{24}$ v3.5.3 (http://www.Rproject.org/). In the boxplots, the upper and lower hinges represented the first and third quartile. The whiskers extended to the most extreme value within 1.5 times the interquartile range on either end of the distribution. The center line represented the median.

\section{REFERENCE}

1. De Coster, W., D'Hert, S., Schultz, D.T., Cruts, M. \& Van Broeckhoven, C. NanoPack: visualizing and processing long-read sequencing data. Bioinformatics 34, 2666-2669 (2018).

2. Li, H. Minimap2: pairwise alignment for nucleotide sequences. Bioinformatics 34, 3094-3100 (2018).

3. Li, H. et al. The Sequence Alignment/Map format and SAMtools. Bioinformatics 25, 2078-9 (2009).

4. Pedersen, B.S. \& Quinlan, A.R. Mosdepth: quick coverage calculation for genomes and exomes. Bioinformatics 34, 867-868 (2018).

5. Beyter, D. et al. Long read sequencing of 1,817 Icelanders provides insight into the role of structural variants in human disease. bioRxiv (2019).

6. Sedlazeck, F.J. et al. Accurate detection of complex structural variations using single-molecule sequencing. Nat Methods (2018).

7. Tham, C.Y. et al. NanoVar: accurate characterization of patients' genomic structural variants using low-depth nanopore sequencing. Genome Bio/21, 56 (2020).

8. Cretu Stancu, M. et al. Mapping and phasing of structural variation in patient genomes using nanopore sequencing. Nat Commun 8, 1326 (2017).

9. Amir Ben-Dor, R.S., and Zohar Yakhini. Clustering Gene Expression Patterns. Journal of Computational Biology 6(1999).

10. Quinlan, A.R. \& Hall, I.M. BEDTools: a flexible suite of utilities for comparing genomic features. Bioinformatics 26, 841-2 (2010).

11. Karolchik, D. et al. The UCSC Table Browser data retrieval tool. Nucleic Acids Res 32, D493-6 (2004).

12. Audano, P.A. et al. Characterizing the Major Structural Variant Alleles of the Human Genome. Cell 176, 663-675 e19 (2019).

13. Pedersen, B.S. et al. Effective variant filtering and expected candidate variant yield in studies of rare human disease. bioRxiv (2020).

14. Hickey, G. et al. Genotyping structural variants in pangenome graphs using the vg toolkit. Genome Bio/ 21, 35 (2020).

15. You, F.M. et al. BatchPrimer3: a high throughput web application for PCR and sequencing primer design. BMC Bioinformatics 9, 253 (2008).

16. MacDonald, J.R., Ziman, R., Yuen, R.K., Feuk, L. \& Scherer, S.W. The Database of Genomic Variants: a curated collection of structural variation in the human genome. Nucleic Acids Res 42, D986-92 (2014).

17. Collins, R.L. et al. gnomAD-SV an open resource of structural variation for medical and population genetics. (2019).

18. Almarri, M.A. et al. Population Structure, Stratification, and Introgression of Human Structural Variation. Cel/ (2020).

19. Kuhn, R.M., Haussler, D. \& Kent, W.J. The UCSC genome browser and associated tools. Brief Bioinform 14, 144-61 (2013).

20. Hubley, R. et al. The Dfam database of repetitive DNA families. Nucleic Acids Res 44, D81-9 (2016).

21. Bao, W., Kojima, K.K. \& Kohany, O. Repbase Update, a database of repetitive elements in eukaryotic 
genomes. Mob DNA 6, 11 (2015).

22. Benson, G. Tandem repeats finder: a program to analyze DNA sequences. Nucleic Acids Research 27, 573-580 (1999).

23. Collins, R.L. et al. A structural variation reference for medical and population genetics. Nature 581, 444-451 (2020).

24. Team, T.R.C. R: A language and environment for statistical computing. R Foundation for Statistical Computing, Vienna, Austria. (2020).

25. Kuleshov, M.V. et al. Enrichr: a comprehensive gene set enrichment analysis web server 2016 update. Nucleic Acids Res 44, W90-7 (2016).

26. Yoav Benjamini, Y.H. Controlling The False Discovery Rate - A Practical And Powerful Approach To Multiple Testing. J. Roy. Stat. Soc. B Met. 57, 289 - 300 (1995).

27. Price, A.L. et al. Principal components analysis corrects for stratification in genome-wide association studies. Nat Genet 38, 904-9 (2006).

28. Bhatia, G., Patterson, N., Sankararaman, S. \& Price, A.L. Estimating and interpreting FST: the impact of rare variants. Genome Res 23, 1514-21 (2013).

29. Asif, H. et al. GWAS significance thresholds for deep phenotyping studies can depend upon minor allele frequencies and sample size. Mol Psychiatry (2020).

30. Purcell, S. et al. PLINK: a tool set for whole-genome association and population-based linkage analyses. Am J Hum Genet 81, 559-75 (2007).

31. Sungwon Jeon, Y.B., Yeonsong Choi, Yeonsu Jeon, Seunghoon Kim, Jaeyoung Jang, Jinho Jang, Asta Blazyte, Changjae Kim, Yeonkyung Kim, Jungae Shim, Nayeong Kim, Yeo Jin Kim, Seung Gu Park, Jungeun Kim, Yun Sung Cho, Yeshin Park, Hak-Min Kim, \& Byoung-Chul Kim, N.-H.P., EunSeok Shin, Byung Chul Kim, Dan Bolser, Andrea Manica, Jeremy S. Edwards, George Church, Semin Lee, Jong Bhak. Korean Genome Project: 1094 Korean personal genomes with clinical information. Sci. Adv., eaaz7835 (2020).

32. Robinson, J.T., Thorvaldsdottir, H., Wenger, A.M., Zehir, A. \& Mesirov, J.P. Variant Review with the Integrative Genomics Viewer. Cancer Res 77, e31-e34 (2017). 


\section{Structural variants in Chinese population and their impact on phenotypes, diseases and population adaptation}

Zhikun Wu, Zehang Jiang, Tong Li, Chuanbo Xie, Liansheng Zhao, Jiaqi Yang, Shuai Ouyang, Yizhi Liu, Tao Li, Zhi Xie

\section{Supplementary Figures and Tables}




\section{Contents}

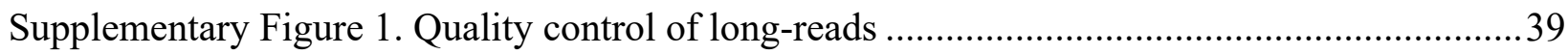

Supplementary Figure 2. Workflow of SV calling and filtering ............................................40

Supplementary Figure 3. SV distribution across meta-chromosome .........................................41

Supplementary Figure 4. Length distribution for DELs and INSs ...........................................42

Supplementary Figure 5. SV numbers of different types in each SV category ...........................43

Supplementary Figure 6. The total number of discovered SVs as a function of number of samples

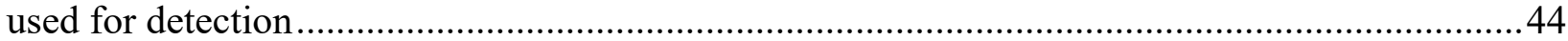

Supplementary Figure 7. GO enrichment analysis for pLoF SVs associated genes.....................45

Supplementary Figure 8. Length distributions of pLoF SVs with different categories................46

Supplementary Table 1. Summary of sample information and sequencing statistics..................47

Supplementary Table 2. Summary of clinical traits................................................................4

Supplementary Table 3. SV statistics of different filtering processes for each sample.................47

Supplementary Table 4. Accumulative lengths of different type SVs for each sample ...............47

Supplementary Table 5. Annotation of repeat sequences in SVs ............................................47

Supplementary Table 6. Summary of PCR validation results of singletons ...............................47

Supplementary Table 7. SVs and associated genes in GWAS, OMIM and COSMIC datasets ....47

Supplementary Table 8. Association for SVs and clinical phenotypes .....................................47

Supplementary Table 9. Information of SVs with significant $F_{\mathrm{st}}(>0.066)$ between Southern and

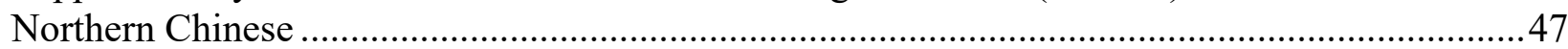


a

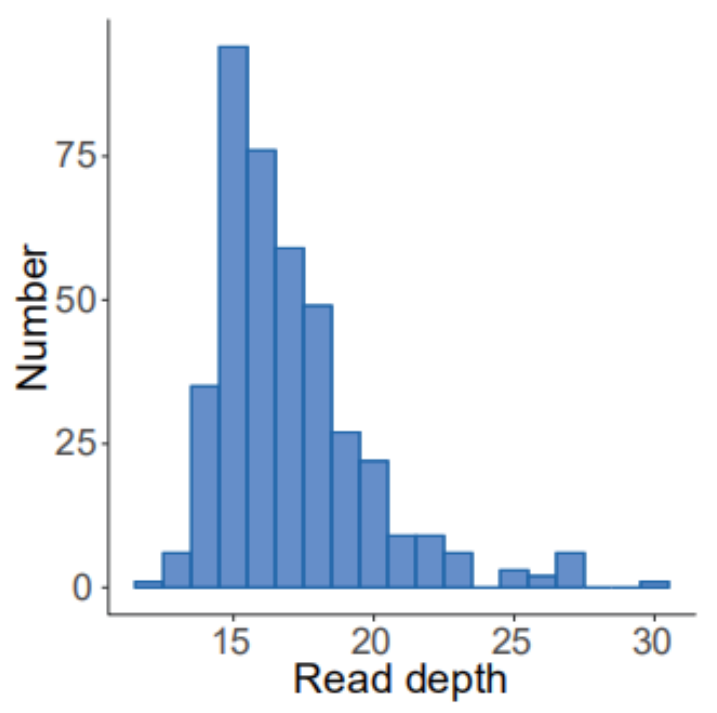

b

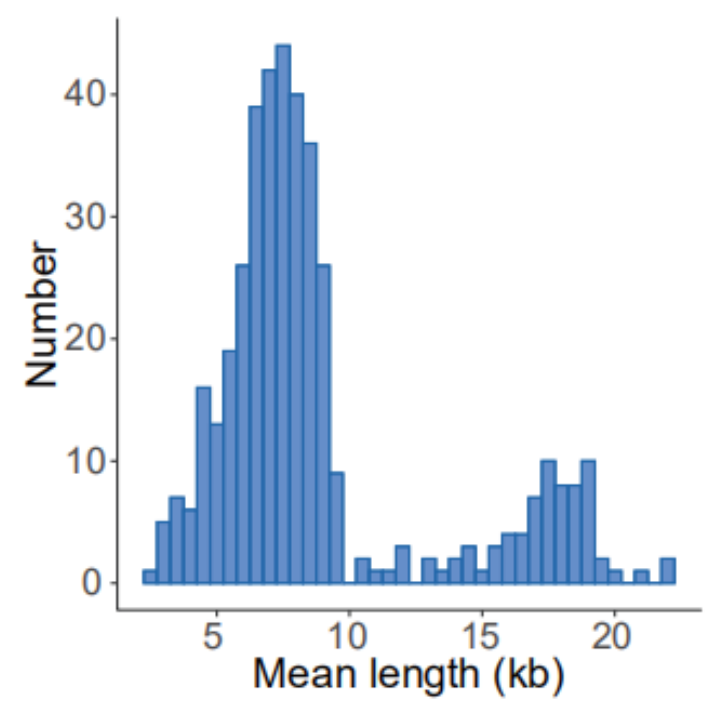

\section{Supplementary Figure 1. Quality control of long-reads}

a, Read depth distribution of clean data for 405 individuals.

b, Distribution of the average read length for 405 individuals. 
a

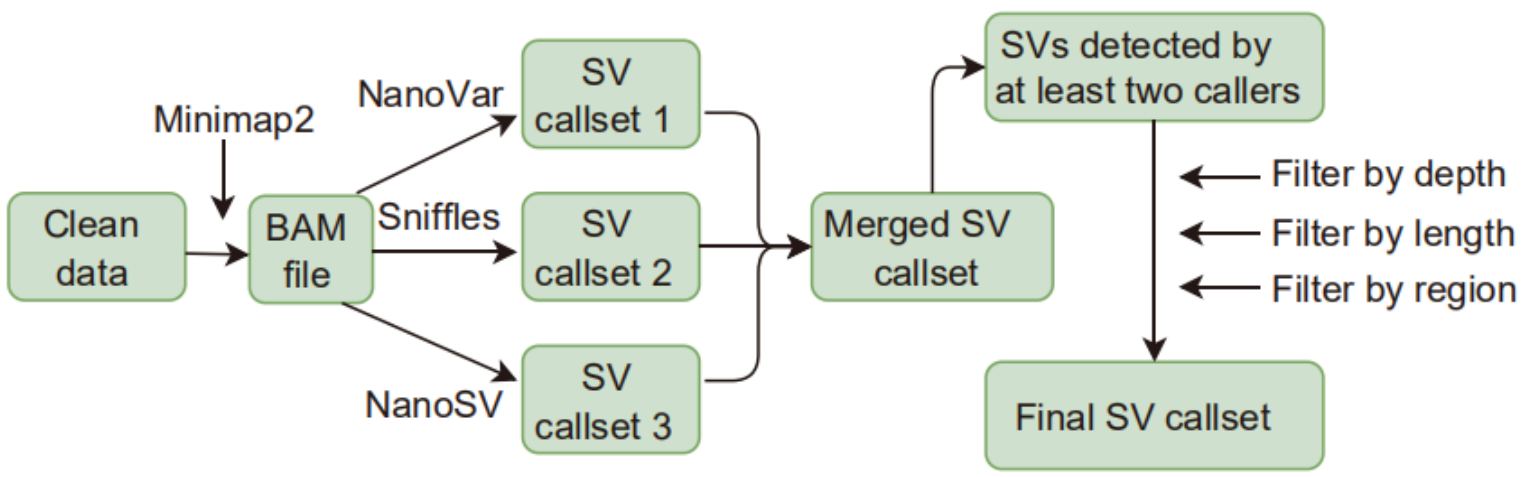

C
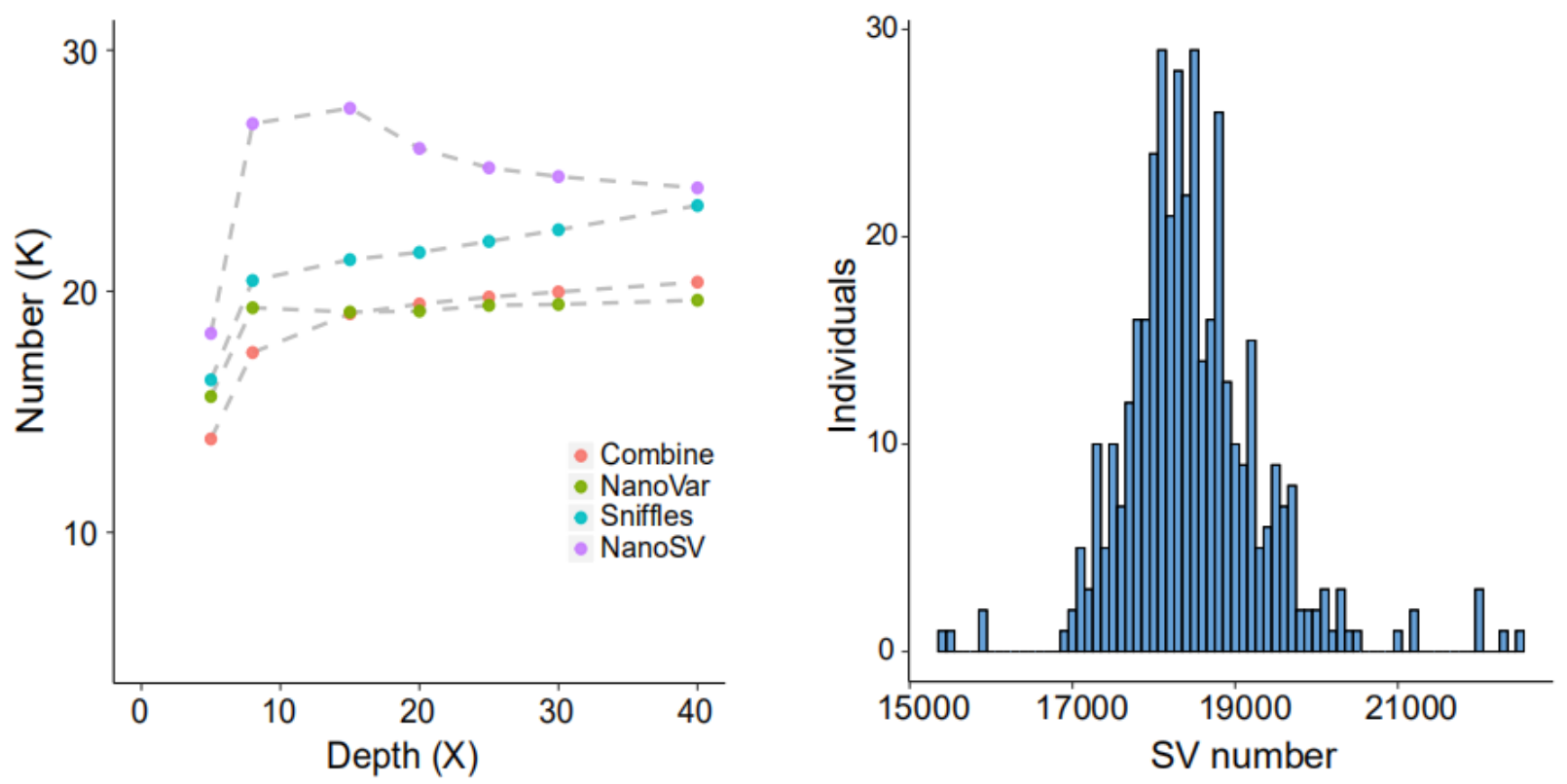

\section{Supplementary Figure 2. Workflow of SV calling and filtering}

a, Workflow of SV calling and filtering for each sample.

b, SV numbers of different callers for reads of different coverages, "Combine" means SVs detected by at least two callers. The threshold of read support of SVs is 0.2 of sequencing depth.

c, Distribution of final high-confidence SVs of all the individuals. 
a

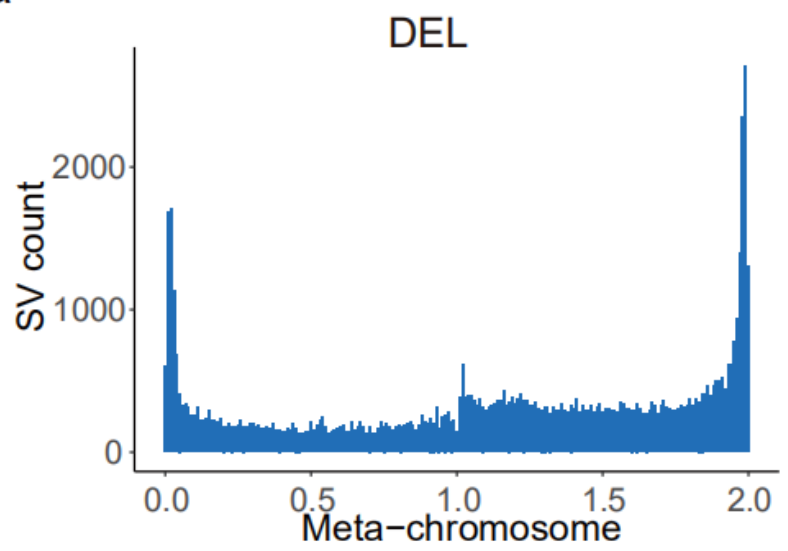

C

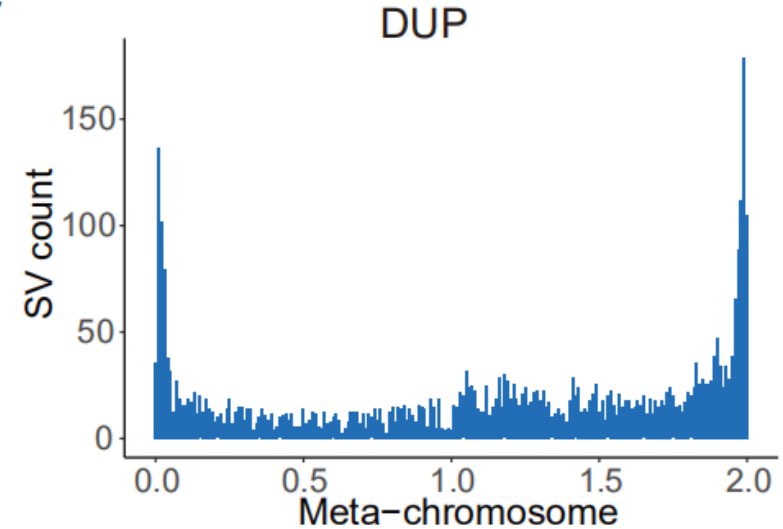

b

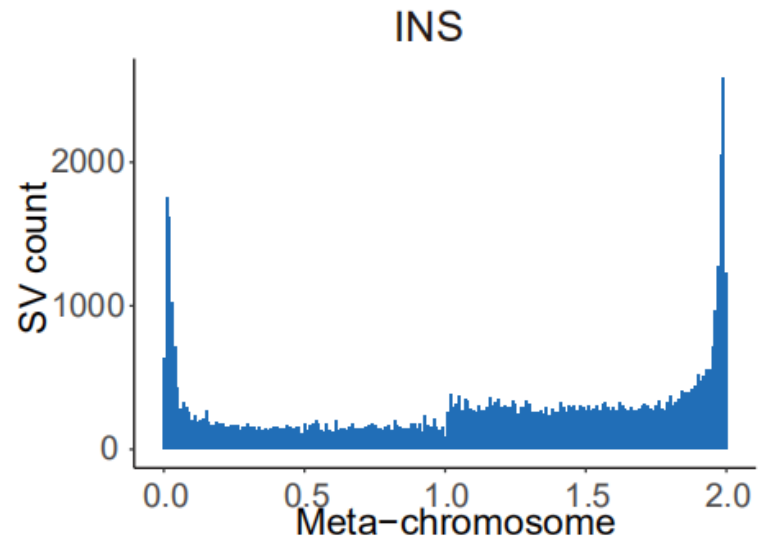

d

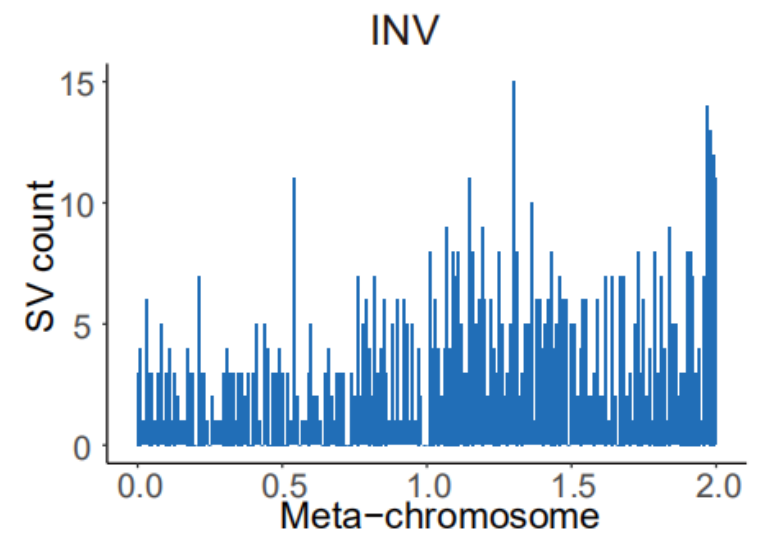

\section{Supplementary Figure 3. SV distribution across meta-chromosome}

SV number across the normalized meta-chromosome for DEL (a), INS (b), DUP (c) and INV (d). 
a

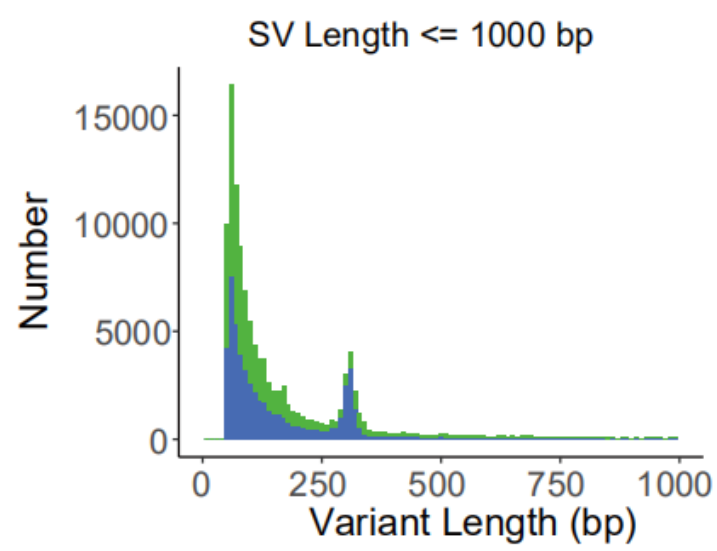

b

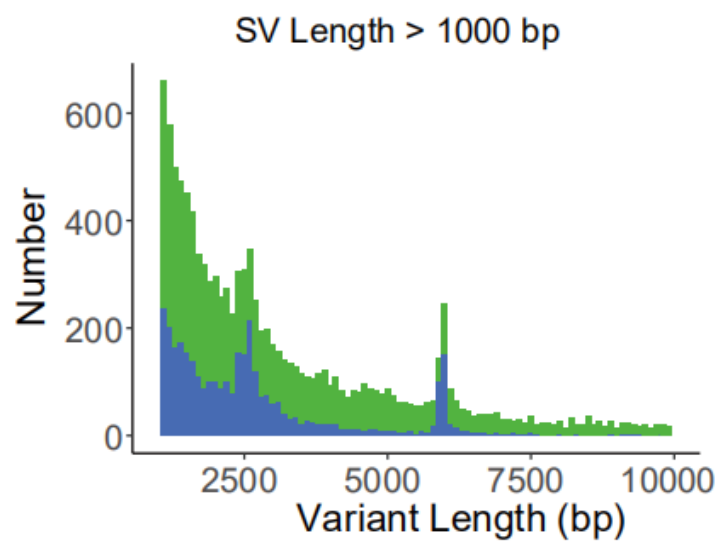

\section{Supplementary Figure 4. Length distribution for DELs and INSs}

SV distribution of DELs and INSs for range of $50 \mathrm{bp}$ to $1 \mathrm{kp}(\mathbf{a})$ and range of $1 \mathrm{~kb}$ to $10 \mathrm{~kb}(\mathbf{b})$.

Two noticeable peaks were observed at sizes around $300 \mathrm{bp}$ and $6 \mathrm{~kb}$. 


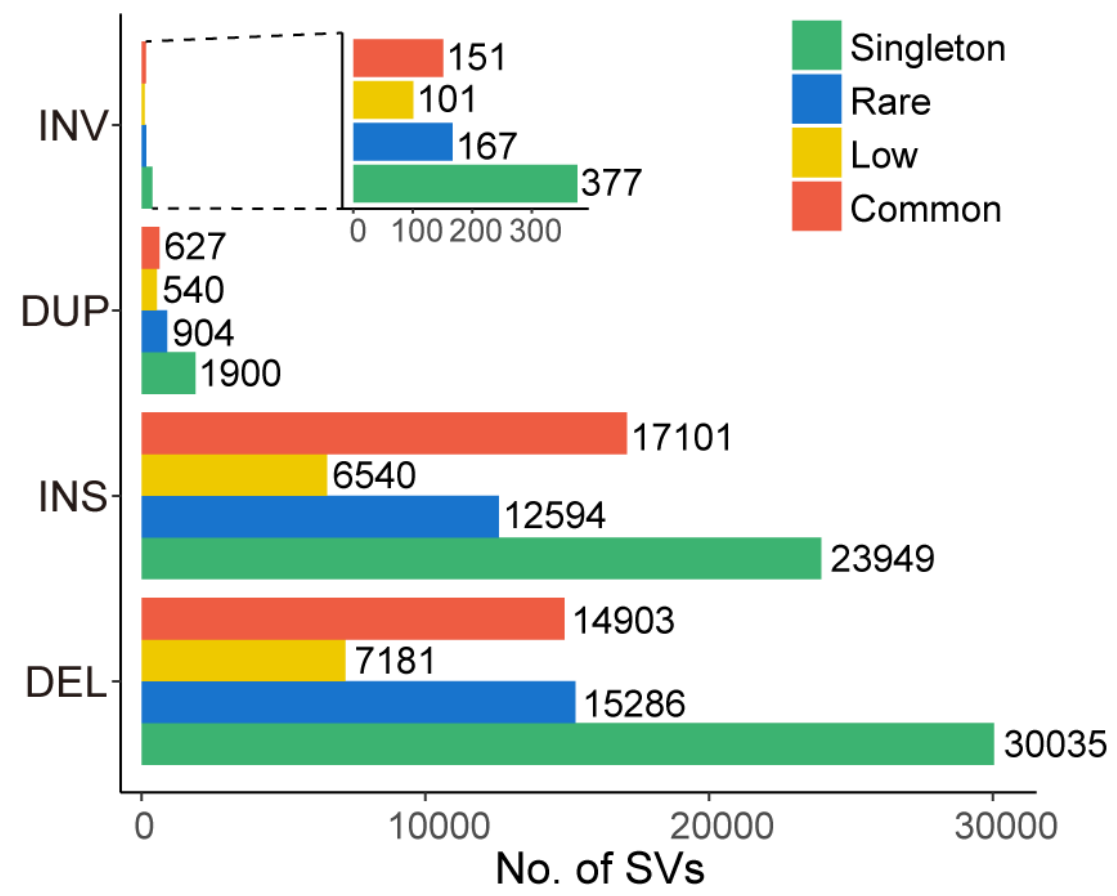

\section{Supplementary Figure 5. SV numbers of different types in each SV category}

SV number for different types of each SV category, the number on the bar graph indicates the actual number of SVs. 


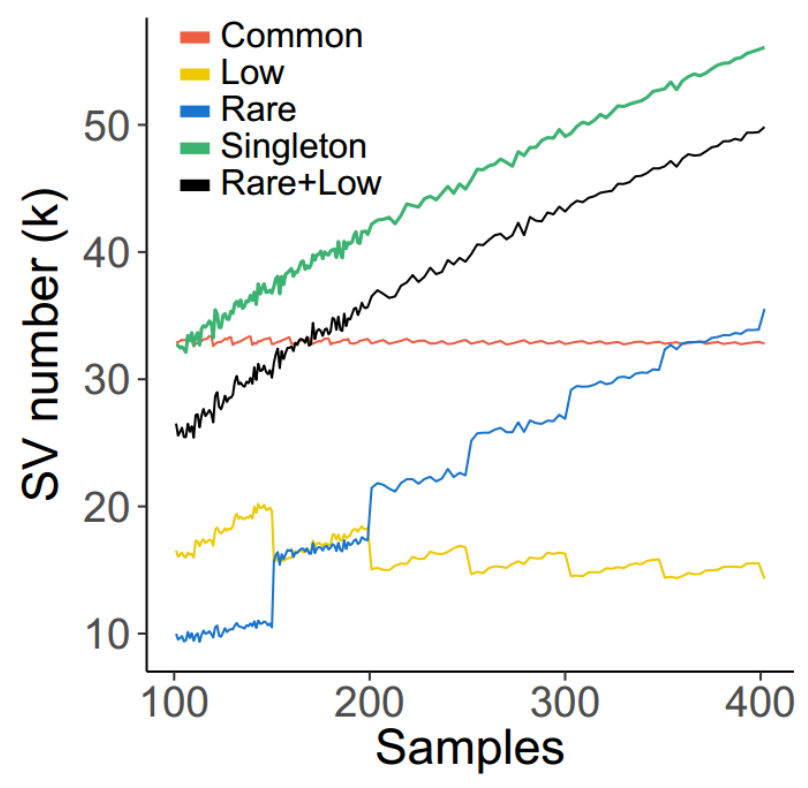

Supplementary Figure 6. The total number of discovered SVs as a function of number of samples used for detection 
bioRxiv preprint doi: https://doi.org/10.1101/2021.02.09.430378; this version posted February 10, 2021. The copyright holder for this preprint (which was not certified by peer review) is the author/funder, who has granted bioRxiv a license to display the preprint in perpetuity. It is made available under aCC-BY-NC-ND 4.0 International license.

Immunoglobulin receptor binding (GO:0034987)

Serine-type endopeptidase activity (GO:0004252)

Serine-type peptidase activity (GO:0008236)

\section{GO (Molecular Function)}

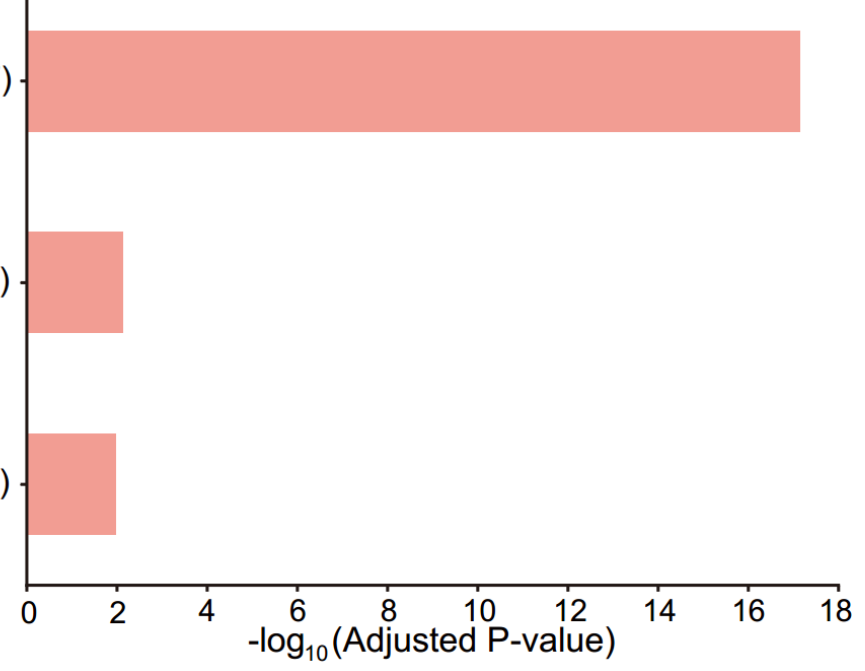

\section{Supplementary Figure 7. GO enrichment analysis for pLoF SVs associated genes}


bioRxiv preprint doi: https://doi.org/10.1101/2021.02.09.430378; this version posted February 10, 2021. The copyright holder for this preprint (which was not certified by peer review) is the author/funder, who has granted bioRxiv a license to display the preprint in perpetuity. It is made available under aCC-BY-NC-ND 4.0 International license.

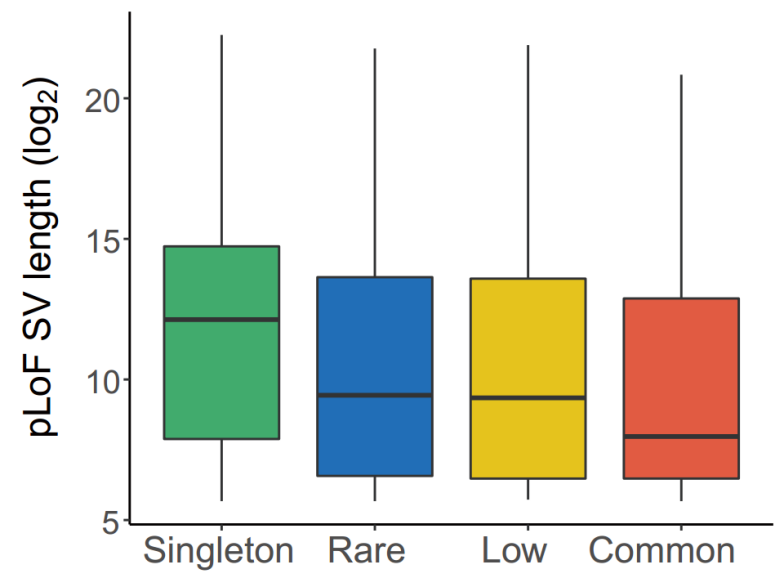

Supplementary Figure 8. Length distributions of pLoF SVs with different categories 
Supplementary Table 1. Summary of sample information and sequencing statistics

Supplementary Table 2. Summary of clinical traits

Supplementary Table 3. SV statistics of different filtering processes for each sample

Supplementary Table 4. Accumulative lengths of different type SVs for each sample

Supplementary Table 5. Annotation of repeat sequences in SVs

Supplementary Table 6. Summary of PCR validation results of singletons

Supplementary Table 7. SVs and associated genes in GWAS, OMIM and

COSMIC datasets

Supplementary Table 8. Association for SVs and clinical phenotypes

Supplementary Table 9. Information of SVs with significant $\boldsymbol{F}_{\text {st }}(>0.066)$

between Southern and Northern Chinese 